\title{
A STUDY OF THE MAIN RESONANCES OUTSIDE THE GEOSTATIONARY RING
}

\author{
ALESSANDRA CELLETTI AND CATALIN GALES
}

\begin{abstract}
We investigate the dynamics of satellites and space debris in external resonances, namely in the region outside the geostationary ring. Precisely, we focus on the 1:2, 1:3, 2:3 resonances, which are located at about $66931.4 \mathrm{~km}, 87705.0 \mathrm{~km}, 55250.7$ $\mathrm{km}$, respectively. Some of these resonances have been already exploited in space missions, like XMM-Newton and Integral.

Our study is mainly based on a Hamiltonian approach, which allows us to get fast and reliable information on the dynamics in the resonant regions. Significative results are obtained even by considering just the effect of the geopotential in the Hamiltonian formulation. For objects (typically space debris) with high area-to-mass ratio the Hamiltonian includes also the effect of the solar radiation pressure. In addition, we perform a comparison with the numerical integration in Cartesian variables, including the geopotential, the gravitational attraction of Sun and Moon, and the solar radiation pressure.

We implement some simple mathematical tools that allows us to get information on the terms which are dominant in the Fourier series expansion of the Hamiltonian around a given resonance, on the amplitude of the resonant islands and on the location of the equilibrium points. We also compute the Fast Lyapunov Indicators, which provide a cartography of the resonant regions, yielding the main dynamical features associated to the external resonances. We apply these techniques to analyze the 1:2, 1:3, 2:3 resonances; we consider also the case of objects with large area-to-mass ratio and we provide an application to the case studies given by XMM-Newton and Integral.
\end{abstract}

\section{INTRODUCTION}

Resonances play a major rôle in the dynamics of satellites around the Earth; indeed, most of the satellites in MEO and GEO regions ${ }^{1}$ are positioned in correspondence with the 2:1 and 1:1 gravitational resonance (see, e.g., [6], [10], [12], [19], [20], [24]). Being in such resonances implies that the satellite makes two orbits or one orbit during one rotation of the Earth around its spin-axis. Other resonances might be important as well, and the aim of this work is to investigate some external resonances, precisely the

\footnotetext{
Key words and phrases. Space debris, Resonance, High area-to-mass ratio.

Corresponding author: E-mail address: celletti@mat.uniroma2.it (Alessandra Celletti).

A.C. was partially supported by the European Grant MC-ITN Stardust, PRIN-MIUR 2010JJ4KPA_009 and GNFM/INdAM.

${ }^{1} \mathrm{MEO}$ and GEO are acronyms for Medium-Earth-Orbit and Geostationary-Earth-Orbit with altitude, respectively, between 2000 and $30000 \mathrm{~km}$ for MEO and larger than $30000 \mathrm{~km}$ for GEO.
} 
1:2, 1:3, 2:3 resonances, whose distances from the center of the Earth are, respectively, about $66931.4 \mathrm{~km}, 87705.0 \mathrm{~km}, 55250.7 \mathrm{~km}$. A remarkable fact is that space missions have already used these resonances; in particular, Integral (International Gamma-Ray Astrophysics Laboratory) has a semimajor axis corresponding to the 1:3 resonance, while the semimajor axis of XMM-Newton (X-ray Multi-Mirror Mission) is at the 1:2 resonance. We claim that such resonances can be particularly useful when dealing with space debris; indeed, the resonant dynamics can be exploited to move space debris in safe regions, either placing them in the stable equilibria, which prevent chaotic variations of semimajor axis, or moving the debris along the chaotic invariant manifold associated to the hyperbolic equilibria.

In order to perform such investigation, we make use of the Hamiltonian formalism, allowing us to make a fast and accurate description of the dynamics. In particular, we introduce a model including a suitable expansion of the geopotential in spherical harmonics. The expansion is limited to a small number of terms, which are chosen by carefully evaluating which of them are the most significative ones in specific regions of the orbital parameter space. In this way, we reduce very much the computational time, although the model still retains the essential features of the dynamics. For objects with high area-to-mass ratio we include also a suitable expansion of the contribution due to the solar radiation pressure. Also in this case we use the Hamiltonian approach.

This strategy allows us to obtain several information on the dynamics within a very short computational time, most notably the location of the equilibrium points, their dependence on the orbital parameters, the size of the resonant regions. Further information are obtained by making a cartography ([8]) of the different regions by means of the computation of the Fast Lyapunov Indicators ([7], see also [1]). In this way we obtain very detailed maps, showing the long term evolution of the semi-major axis, although within the Hamiltonian approach we limit the cartography to a model including just the geopotential and, possibly, the solar radiation pressure. As a consequence, to validate our results we make a comparison with the Cartesian equations of motion by computing maps which include also the effects of Sun and Moon. Attention must be paid in comparing the results and, precisely, while integrating the Cartesian equations of motion, we need to transform from osculating to mean orbital elements.

We also provide an application of our technique to two sample cases: XMM-Newton, which is related to the 1:2 resonance, and Integral, related to the 1:3 resonance. These 
space missions are characterized by a large eccentricity; henceforth, a dedicated expansion of the geopotential is necessary, in order to include terms which are relevant at high eccentricities. Again, we use the Cartesian approach to validate the results obtained through the Hamiltonian formalism, although we find that in the case of XMM-Newton the effect of the Moon shapes the resonant islands and should be included in the overall discussion to obtain an accurate description of the dynamics.

This paper is organized as follows. In Section 2 we introduce the Cartesian equations of motion, including the geopotential, the influence of Sun and Moon, and the solar radiation pressure. In Section 3 we introduce the Hamiltonian of the geopotential and we provide explicit expressions for the secular and resonant expansions of the Hamiltonian. In Section 4 we make a qualitative analysis of the resonant regions, by reducing the study to a very limited number of terms of the expansion and by using the pendulumlike structure of the resonant zone to compute the amplitude of the resonant islands. A cartography based on the computation of the Fast Lyapunov Indicators is given in Section 5, while the case of large area-to-mass ratio is investigated in Section 6 . The cases of XMM-Newton and Integral are studied in Section 7, where the expansions of the geopotential have been extended to encompass the case of large eccentricities. Some conclusions and perspectives are presented in Section 8.

\section{Cartesian and Hamiltonian equations of motion}

We consider a small body, say $S$, moving in the gravitational field of the Earth, which we assume to be oblate, and subject to the influence of Sun, Moon and to the effect of the solar radiation pressure (hereafter SRP). We assume that the body is so small, that its influence on Earth, Sun and Moon can be neglected. Let $\mathbf{r}=(x, y, z)$ be the radius vector of $S$ in a geocentric quasi-inertial fixed frame.

The corresponding equations of motion are the sum of the equations describing the Earth's gravitational influence, the oblateness effect, the solar and lunar attraction, and the SRP. Let $m_{S}, m_{M}$ be the masses of Sun and Moon, $\mathbf{r}_{S}, \mathbf{r}_{M}$ the position vectors of Sun and Moon (whose explicit expressions are given, e.g., in [18]), $\mathcal{G}$ the gravitational constant. The equations of motion of $S$ can be written as

$$
\begin{aligned}
\ddot{\mathbf{r}} & =R_{3}(-\theta) \nabla V(\mathbf{r})-\mathcal{G} m_{S}\left(\frac{\mathbf{r}-\mathbf{r}_{S}}{\left|\mathbf{r}-\mathbf{r}_{S}\right|^{3}}+\frac{\mathbf{r}_{S}}{\left|\mathbf{r}_{S}\right|^{3}}\right) \\
& -\mathcal{G} m_{M}\left(\frac{\mathbf{r}-\mathbf{r}_{M}}{\left|\mathbf{r}-\mathbf{r}_{M}\right|^{3}}+\frac{\mathbf{r}_{M}}{\left|\mathbf{r}_{M}\right|^{3}}\right)+C_{r} P_{r} a_{S}^{2}\left(\frac{A}{m}\right) \frac{\mathbf{r}-\mathbf{r}_{S}}{\left|\mathbf{r}-\mathbf{r}_{S}\right|^{3}},
\end{aligned}
$$


where $R_{3}$ is the rotation matrix about the Earth's polar axis, $\theta$ is the sidereal time, $\nabla$ is the gradient in the synodic frame, $V(\mathbf{r})$ is the force function due to the attraction of the Earth:

$$
V(\mathbf{r})=\mathcal{G} \int_{V_{E}} \frac{\rho\left(\mathbf{r}_{p}\right)}{\left|\mathbf{r}-\mathbf{r}_{p}\right|} d V_{E}
$$

where $\rho\left(\mathbf{r}_{p}\right)$ is the density at some point $\mathbf{r}_{p}$ in the Earth and $V_{E}$ is the volume of the Earth. The second and third terms in (2.1) model the attraction of Sun and Moon, respectively. The last term in (2.1) models the SRP and depends on the adimensional reflectivity coefficient $C_{r}$ (fixed to 1 in this paper), the radiation pressure $P_{r}=4.56 \cdot 10^{-6} \mathrm{~N} / \mathrm{m}^{2}$ for a body located at $a_{S}=1 \mathrm{AU}$, the area-to-mass ratio $A / m$ with $A$ the cross-section of $S$ and $m$ its mass.

We proceed now to write in Hamiltonian formulation the term in (2.1) corresponding to the geopotential. To this end, we introduce the action-angle Delaunay variables, denoted as $(L, G, H, M, \omega, \Omega)$, where $L=\sqrt{\mu_{E} a}, G=L \sqrt{1-e^{2}}, H=G \cos i$ with $a$ the semimajor axis, $e$ the eccentricity, $i$ the inclination, $M$ the mean anomaly, $\omega$ the argument of perigee, $\Omega$ the longitude of the ascending node and $\mu_{E}=\mathcal{G} m_{E}$ with $m_{E}$ the mass of the Earth. The geopotential Hamiltonian can then be written as (see [2])

$$
\mathcal{H}(L, G, H, M, \omega, \Omega, \theta)=-\frac{\mu_{E}^{2}}{2 L^{2}}+R_{e a r t h}(L, G, H, M, \omega, \Omega, \theta)
$$

where $R_{\text {earth }}$ represents the perturbing function, whose explicit expression is given as follows.

In the geocentric quasi-inertial frame, the geopotential in (2.2) can be expanded as $([11])$

$$
R_{\text {earth }}=-\frac{\mu_{E}}{a} \sum_{n=2}^{\infty} \sum_{m=0}^{n}\left(\frac{R_{E}}{a}\right)^{n} \sum_{p=0}^{n} F_{n m p}(i) \sum_{q=-\infty}^{\infty} G_{n p q}(e) S_{n m p q}(M, \omega, \Omega, \theta)
$$

where the inclination and eccentricity functions $F_{n m p}, G_{n p q}$ can be computed by wellknown recursive formulae (see, e.g., [11]). The angle $S_{n m p q}$ is defined as

$$
S_{n m p q}=\left[\begin{array}{c}
C_{n m} \\
-S_{n m}
\end{array}\right]_{n-m \text { odd }}^{n-m \text { even }} \cos \Psi_{n m p q}+\left[\begin{array}{c}
S_{n m} \\
C_{n m}
\end{array}\right]_{n-m \text { odd }}^{n-m \text { even }} \sin \Psi_{n m p q},
$$

where $C_{n m}$ and $S_{n m}$ are, respectively, the cosine and sine coefficients of the spherical harmonics potential terms (see Table 1 for concrete values) and

$$
\Psi_{n m p q}=(n-2 p) \omega+(n-2 p+q) M+m(\Omega-\theta) .
$$


As common in geodesy, we introduce also the quantities $J_{n m}$ defined by

$$
J_{n m}=\sqrt{C_{n m}^{2}+S_{n m}^{2}} \text { if } m \neq 0, \quad J_{n 0} \equiv J_{n}=-C_{n 0}
$$

and the quantities $\lambda_{n m}$ defined through the relations

$$
C_{n m}=-J_{n m} \cos \left(m \lambda_{n m}\right), \quad S_{n m}=-J_{n m} \sin \left(m \lambda_{n m}\right)
$$

\section{SeCular And Resonant Hamiltonian}

The expansion of the function $R_{\text {earth }}$ in (2.3) contains an infinite number of trigonometric terms, but the long term variation of the dynamics is mainly governed by the secular and resonant terms. Let us recall that a gravitational resonance (also called a tesseral resonance) occurs whenever there is a commensurability between the orbital period of the minor body and the period of rotation of the Earth.

In astronomical applications the condition of gravitational resonance is satisfied only within a certain approximation and cannot be obviously satisfied exactly.

Notice that, by using Kepler's third law, a $j: \ell$ resonance corresponds to a semimajor axis equal to $a_{j: \ell}=(j / \ell)^{-2 / 3} a_{g e o}$, where $a_{g e o}=42164.1696 \mathrm{~km}$ is the semimajor axis corresponding to the geostationary orbit. From this formula we derive the location of the $1: 2$ resonance at $66931.4 \mathrm{~km}$, the $1: 3$ resonance is found at $87705.0 \mathrm{~km}$, while the $2: 3$ resonance is at $55250.7 \mathrm{~km}$.

Let us expand the Earth's gravitational potential up to terms of degree and order $n=m=N$ for some $N>0$; then, we approximate $R_{\text {earth }}$ with the expression

$$
R_{\text {earth }}=R_{\text {earth }}^{\text {sec }}+R_{\text {earth }}^{\text {res }}+R_{\text {earth }}^{\text {nonres }} \cong \sum_{n=2}^{N} \sum_{m=0}^{n} \sum_{p=0}^{n} \sum_{q=-\infty}^{\infty} \mathcal{T}_{n m p q}
$$

where $R_{\text {earth }}^{\text {sec }}, R_{\text {earth }}^{\text {res }}, R_{\text {earth }}^{\text {nonres }}$ denote, respectively, the secular, resonant and non-resonant contributions to the Earth's potential and where we have introduced the coefficients:

$$
\mathcal{T}_{n m p q}=-\frac{\mu_{E} R_{E}^{n}}{a^{n+1}} F_{n m p}(i) G_{n p q}(e) S_{n m p q}(M, \omega, \Omega, \theta)
$$

Remark 1. From (2.5) we see that the quantity $\dot{\Psi}_{\text {nmpq }}$ depends also on $\dot{\omega}, \dot{\Omega}$, which can be small, but not exactly zero. As a consequence, the stationary solutions have different locations according to the values of the integers $n, m, p, q$. As already noticed in [2], this implies that each resonance might split into a multiplet of resonances. 
Our next task will consist in the determination of the secular part of the expansion (2.3) by computing the average over the fast angles, say $R_{\text {earth }}^{\text {sec }}$, and the determination of the resonant part associated to a given $j: \ell$ gravitational resonance, say $R_{\text {earth }}^{\text {res }} \ell$.

Since the value of the oblateness coefficient $J_{2}=J_{20}$ is much larger than the value of any other zonal coefficient (see Table 1), we consider the same secular part for all resonances; the explicit expression will be given in Section 3.1 and Appendix A.

\begin{tabular}{|c|c|c|c|c|c|}
\hline$n$ & $m$ & $C_{n m}$ & $S_{n m}$ & $J_{n m}$ & $\lambda_{n m}$ \\
\hline 2 & 0 & -1082.6261 & 0 & 1082.6261 & 0 \\
2 & 1 & -0.000267 & 0.0017873 & 0.001807 & $-81^{\circ} .5116$ \\
2 & 2 & 1.57462 & -0.90387 & 1.81559 & $75^{\circ} .0715$ \\
3 & 0 & 2.53241 & 0 & -2.53241 & 0 \\
3 & 1 & 2.19315 & 0.268087 & 2.20947 & $186^{\circ} .9692$ \\
3 & 2 & 0.30904 & -0.211431 & 0.37445 & $72^{\circ} .8111$ \\
4 & 0 & 1.6199 & 0 & -1.619331 & 0 \\
4 & 1 & -0.50864 & -0.449265 & 0.67864 & $41^{\circ} .4529$ \\
4 & 2 & 0.078374 & 0.148135 & 0.16759 & $121^{\circ} 0589$ \\
4 & 3 & 0.059215 & -0.012009 & 0.060421 & $56^{\circ} .1784$ \\
\hline
\end{tabular}

TABLE 1 . The coefficients $C_{n m}, S_{n m}, J_{n m}$ (in units of $10^{-6}$ ) up to degree and order 5; values computed from [5] (see also [3], [18]).

The expansions of the resonant part, say $R_{\text {earth }}^{\text {res } j: \ell}$, are composed by several different terms, say $\mathcal{T}_{k}$ for some $k \in \mathbb{Z}_{+}$; in practical computations it is essential to retain the minimum number of significant terms. To this end, we introduce the following heuristic definition of dominant term.

Definition 2. Consider a $j: \ell$ gravitational resonance and let $\lambda^{(j \ell)}$ be the associated stroboscopic mean node (see [9], [14]). Given the orbital elements ( $a, e, i)$, we say that a term $\mathfrak{T}_{k}$ for some $k \in \mathbb{Z}_{+}$of the expansion of $R_{\text {earth }}^{\text {res } j: \ell}$ say $\mathfrak{T}_{k}=g_{k}(a, e, i) \cos \left(k \lambda^{(j \ell)}+\right.$ $\gamma_{k}$ ) for some $\gamma_{k}$ constant, is dominant with respect to the other harmonic terms of the resonant part, if the size of $\left|g_{k}(a, e, i)\right|$ is bigger than the size of any other term of the expansion.

Making use of Definition 2, for a given $j: \ell$ gravitational resonance, we proceed to approximate the Hamiltonian function by the expression

$$
\mathcal{H}^{\text {res } j: \ell}=-\frac{\mu_{E}^{2}}{2 L^{2}}+R_{\text {earth }}^{\text {sec }}+R_{\text {earth }}^{\text {res } j, \ell}
$$


where for the resonant part $R_{\text {earth }}^{r e s j: \ell}$ we considered an optimal degree $N$ of the expansion, obtained using the following algorithm:

$i$ ) expand the resonant part up to a degree $n$ large enough (at least $n=N+1$ );

ii) plot the dominant terms of the expansion as a function of $e, i$ within prescribed intervals that we choose to be $e \in[0,0.5], i \in\left[0,90^{\circ}\right]$;

iii) compute the size of each function $\left|g_{k}\left(a_{j: \ell}, e, i\right)\right|$;

$i v$ ) through a color scale represent the index $k$ of the dominant term in the plane $e-i$,

$v$ ) the optimal degree $N$ of the expansion is the largest degree of the terms, whose index is represented in the plot of dominant terms computed in $i v$ ).

For the 1:2 and 1:3 resonances the optimal degree of expansion of $R_{\text {earth }}^{\text {res } j: \ell}$ is $N=4$, while for the 2:3 resonance the optimal degree is 3 . In the next sections we provide the secular and resonant terms up to the second order in the eccentricity; explicit expressions of all coefficients providing the secular and resonant terms are given in Appendix A.

3.1. The secular part of $R_{\text {earth }}$. With reference to the expression for $S_{n m p q}$ given in (2.4)-(2.5), the secular terms correspond to $m=0$ and $n-2 p+q=0$. We consider the secular part up to terms of degree $n=4$ and neglect higher order harmonic terms.

Since $G_{n p q}(e)=\mathcal{O}\left(e^{|q|}\right)$, then up to second order in the eccentricity, the secular part is given by

$$
R_{\text {earth }}^{\text {sec }} \cong \mathcal{T}_{200-2}+\mathcal{T}_{2010}+\mathcal{T}_{2022}+\mathcal{T}_{301-1}+\mathcal{T}_{3021}+\mathcal{T}_{401-2}+\mathcal{T}_{4020}+\mathcal{T}_{4032},
$$

where the functions $\mathcal{T}_{n m p q}$ have been introduced in (3.1). Using the formulae given by [11] for the functions $F_{n m p}$ and $G_{n p q}$, the explicit expression of the above terms can be found in Appendix A.

3.2. The resonant part of $R_{\text {earth }}$. From (2.4)-(2.5) we see that the terms associated to a resonance of order $j: \ell$ correspond to $j(n-2 p+q)=\ell m$. We consider the resonant part up to degree and order $n=m=N$ with $N=4$ for the 1:2, 1:3 resonances and $N=3$ for the $2: 3$ resonance. The terms whose sum provides the resonant part of $R_{\text {earth }}^{\text {resj: }}$ for the 1:2, 1:3, 2:3 resonances are listed in Table 2, while their explicit expressions are given in Appendix A.

Remark 3. Except for the 1:3 resonance, the expansions up to the second order in the eccentricity of $R_{\text {earth }}^{\text {resj: }}$ describe with a good enough accuracy the main dynamical features 


\begin{tabular}{|c|c|c|}
\hline$j: \ell$ & $N$ & terms \\
\hline $1: 2$ & 4 & $\mathcal{T}_{2100}, \mathcal{T}_{2112}, \mathcal{T}_{2202}, \mathcal{T}_{310-1}, \mathcal{T}_{3111}, \mathcal{T}_{3201}, \mathcal{T}_{410-2}, \mathcal{T}_{4110}, \mathcal{T}_{4122}, \mathcal{T}_{4200}, \mathcal{T}_{4212}, \mathcal{T}_{4302}$ \\
\hline $1: 3$ & 4 & $\mathcal{T}_{2101}, \mathcal{T}_{2204}, \mathcal{T}_{3100}, \mathcal{T}_{3112}, \mathcal{T}_{410-1}, \mathcal{T}_{4111}, \mathcal{T}_{4202}$ \\
\hline $2: 3$ & 3 & $\mathcal{T}_{2201}, \mathcal{T}_{3200}, \mathcal{T}_{3212}$ \\
\hline
\end{tabular}

TABLE 2. Terms whose sum provides $R_{\text {earth }}^{\text {resj: }}$; the explicit expressions are given in Appendix A.

of the resonances when the eccentricity is in the range $e \in[0,0.5]$. However, for the 1:3 resonance, there is a term of order four in the eccentricity, precisely $\mathcal{T}_{2204}$, which plays a very important rôle for moderate and large eccentricities (see Section 5.3).

\section{Qualitative AnAlysis}

Following [2], making use of elementary mathematical methods, we perform a first order qualitative analysis to select the dominant terms in specific regions of the parameter space (Section 4.1). After this selection, we proceed to give an estimate of the amplitude of the resonant islands (Section 4.2). This strategy will allow us to obtain useful information on the dynamics of the resonances within a very limited computational time.

4.1. Dominant terms. Starting from the series expansions provided in Sections 3.1 and 3.2, for each value of $e, i$, we compute the dominant terms according to Definition 2. Hence, we obtain the plots for the 1:2, 1:3, 2:3 resonances, as shown in Figure 1, upper panels. The orbital elements $(e, i)$ are taken within the intervals $e \in[0,0.5], i \in\left[0^{\circ}, 90^{\circ}\right]$.

Since the magnitude of the resonant terms decays with the degree $n$ as $\left(R_{E} / a\right)^{n}$, and since the semimajor axis is very large for the considered resonances, the most relevant rôle is played by the harmonic terms of order $\mathcal{O}\left(J_{22}\right)$, even though they are of high order in the eccentricity. From Table 3 we see that we are led to consider at most 5 terms, thus reducing considerably the expansions for a specific resonance.

\begin{tabular}{|c|c|c|c|c|c|}
\hline Resonance & Black & Yellow & Brown & Blue & White \\
\hline $1: 2$ & $\mathcal{T}_{2202}$ & $\mathcal{T}_{4110}$ & $\mathcal{T}_{3201}$ & $\mathcal{T}_{3111}$ & $\mathcal{T}_{4200}$ \\
\hline $1: 3$ & $\mathcal{T}_{2204}$ & $\mathcal{T}_{3100}$ & $\mathcal{T}_{3112}$ & - & - \\
\hline $2: 3$ & $\mathcal{T}_{2201}$ & $\mathcal{T}_{3200}$ & - & - & - \\
\hline
\end{tabular}

TABLE 3. The colors used in Figure 1 to represent the dominant terms. 

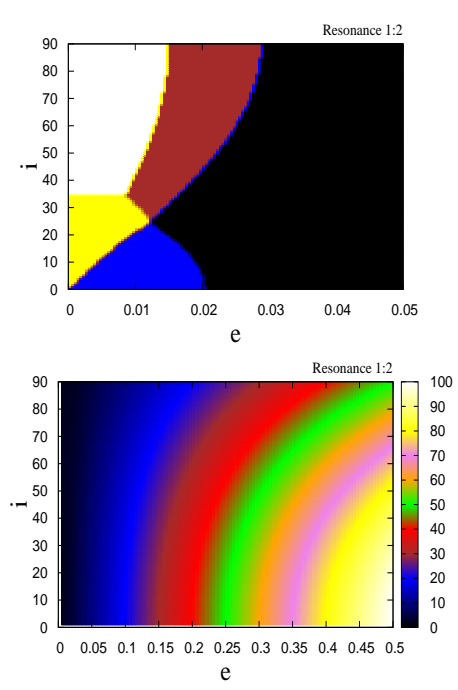
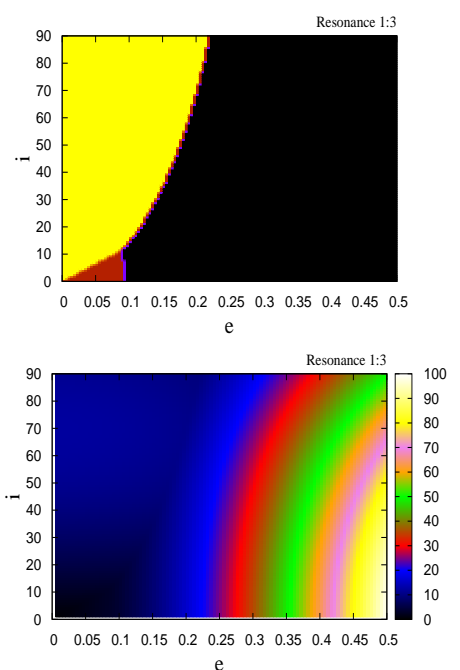
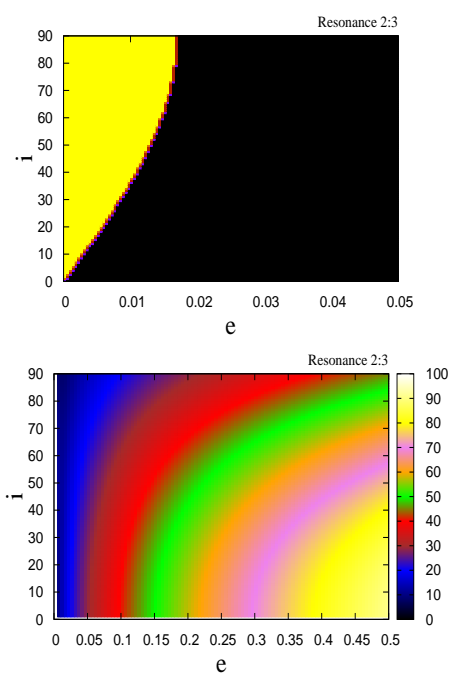

Figure 1. Left 1:2, center 1:3, right 2:3 resonance. Upper plots: dominant terms; for the 1:2 and 2:3 resonances we magnified the region $[0,0.05] \times\left[0^{\circ}, 90^{\circ}\right]$, because in the rest of the domain the color is black.

The colors used to represent the dominant terms are explained in Table 3. Lower plots: amplitude of the resonances for $\omega=0^{\circ}, \Omega=0^{\circ}$; the color bar provides the measure of the amplitude in kilometers.

4.2. Amplitude of the resonant islands. The amplitude of the island around a given $j$ : $\ell$ resonance is obtained by making an expansion of the Hamiltonian around the resonance, thus reducing the problem to a pendulum-like Hamiltonian. This expansion will provide an analytical estimate of the amplitude of the resonant region (we refer to [2] for full details). Let us briefly summarize the method devised in [2]. We start by taking into account the secular part and, precisely, just the largest term of the resonant part. Let the resonant Hamiltonian be written as

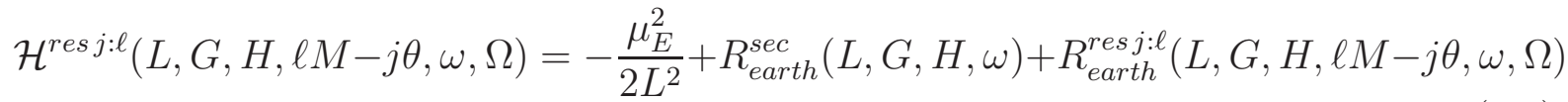

with

$R_{\text {earth }}^{\text {res } j \ell}(L, G, H, \ell M-j \theta, \omega, \Omega) \equiv \sum_{k_{1}=1}^{N_{1}} \sum_{k_{2}=1}^{N_{2}} \sum_{k_{3}=1}^{N_{3}} R_{\underline{k}}^{(j, \ell)}(L, G, H) c s\left(k_{1}(\ell M-j \theta)+k_{2} \omega+k_{3} \Omega\right)$

for some integers $N_{1}, N_{2}, N_{3}$ and for some Fourier coefficients $R_{\underline{k}}^{(j, \ell)}$. In the above relation, cs could be either cosine or sine and $\underline{k}=\left(k_{1}, k_{2}, k_{3}\right)$. Let $L=L_{\text {res }}$ be the resonant value of 
the action $L$, which can be evaluated through Kepler's third law. Expanding (4.1) around $L_{\text {res }}$ up to second order and retaining only the largest term in the resonant Hamiltonian, we obtain a Hamiltonian of the form

$$
\begin{aligned}
\mathcal{H}_{\max }^{\text {res } j \ell}(L, G, H, \ell M-j \theta, \omega, \Omega) & =\alpha\left(L-L_{\text {res }}\right)-\beta\left(L-L_{\text {res }}\right)^{2} \\
& +\gamma c s\left(k_{1}^{\max }(\ell M-j \theta)+k_{2}^{\max } \omega+k_{3}^{\max } \Omega\right),
\end{aligned}
$$

for suitable functions which can be approximated as $\alpha \simeq \mu_{E}^{2} / L_{r e s}^{3}, \beta \simeq 3 \mu_{E}^{2} /\left(2 L_{r e s}^{4}\right)$, while $\gamma$ is the largest coefficient, say $\gamma=R_{\underline{k}_{\max }^{(j, \ell)}}\left(L_{r e s}, G, H\right)$ with index $\underline{k}_{\max }=\left(k_{1}^{\max }, k_{2}^{\max }, k_{3}^{\max }\right)$. One can show (see [2]) that the amplitude of the $j: \ell$ resonant island is given by

$$
2 \Delta a=\frac{2}{\mu_{E}}\left(\frac{2 \gamma}{\beta}+2 L_{\text {res }} \sqrt{\frac{2 \gamma}{\beta}}\right) .
$$

We report in Figure 1 (lower panels) the amplitudes of the 1:2, 1:3, 2:3 resonances as a function of the eccentricity (between 0 and 0.5 ) and the inclination (between $0^{\circ}$ and $90^{\circ}$ ); in the computations we fixed $\omega=0^{\circ}$ and $\Omega=0^{\circ}$. The color bar provides the size of the amplitude in kilometers. A comparison with the results of Section 5 shows a remarkable agreement between these figures and the results we will obtain plotting the FLIs (using a much longer computational time).

4.3. Casting the results. In this section we collect the information based on the following items, which have been investigated in the previous sections:

(1) the dominant terms with reference to Figure 1, which provide the terms of the expansions in Sections 3.1 and 3.2 leading the dynamics (see Section 4.1);

(2) the amplitude of the resonances associated to each dominant term (see Section 4.2);

(3) the location of the equilibrium points associated to the different dominant terms, according to the expansion in Section 4.2 (see also Appendix A).

This analysis has several advantages: $(i)$ it is based on analytical arguments, $(i i)$ it does not need to integrate the equations of motion, (iii) it can be carried out within a very limited computer time. Indeed, the following information will provide a fast tool to obtain a qualitative behavior, which will be confirmed and refined by the cartographic study performed in Section 5 .

1:2 resonance. The dominant terms are $\mathcal{T}_{2202}, \mathcal{T}_{3111}, \mathcal{T}_{3201}, \mathcal{T}_{4110}, \mathcal{T}_{4200}$, but the most important one is $\mathcal{T}_{2202}$, which corresponds to the biggest amplitude of a few tenths of kilometers in the region in which $e \geq 0.1$. Since the trigonometric argument of $\mathcal{T}_{2202}$ 
is $2\left(\sigma_{12}-\omega-\lambda_{22}\right)$ (see Appendix A), one gets in the $\left(\sigma_{12}, a\right)$ plane a single resonant island in an interval of length of $180^{\circ}$ on the $\sigma_{12}$ axis, with the stable point located at $\sigma_{12}=\lambda_{22}+\omega+180^{\circ} \kappa, \kappa \in \mathbb{Z}$. In general, the amplitude of the resonant island is bigger as the eccentricity is larger and the inclination is smaller.

All these information are consistent with those shown in Figure 2; in fact, the equilibrium is located at about $\lambda_{22} \simeq 75^{\circ}$ (see Table 1 ), while the amplitude is in agreement with the lower plots of Figure 1.

1:3 resonance. The dominant terms are $\mathcal{T}_{2204}, \mathcal{T}_{3100}, \mathcal{T}_{3112}$. Although $\mathcal{T}_{2204}$ is of order four in the eccentricity, it is dominant for a large region of the $(e, i)$-plane. As a consequence, for moderate and large eccentricities the stable equilibrium points are located at $\sigma_{13}=\lambda_{22}+2 \omega+180^{\circ} \kappa, \kappa \in \mathbb{Z}$. On the contrary, for small eccentricities and nonzero inclinations, $\mathcal{T}_{3100}$ is dominant and therefore the elliptic equilibrium points are located at $\sigma_{13}=\lambda_{31}-180^{\circ}+360^{\circ} \kappa, \kappa \in \mathbb{Z}$.

Again, we find a very good agreement with the results of Section 5 (see, e.g., Figure 3).

2:3 resonance. The dominant terms are $\mathcal{T}_{2201}, \mathcal{T}_{3200}$; the term $\mathcal{T}_{3200}$ is dominant for small eccentricities (say $e \leq 0.02$ ). The term $\mathcal{T}_{2201}$ is dominant almost everywhere else. The width of the resonance is bigger as the eccentricity is larger. The stable equilibrium points are located at $\sigma_{23}=2 \lambda_{22}+\omega+360^{\circ} \kappa, \kappa \in \mathbb{Z}$, when $\mathcal{T}_{2201}$ is dominant, while they are at $\sigma_{23}=2 \lambda_{32}+90^{\circ}+360^{\circ} \kappa, \kappa \in \mathbb{Z}$, when $\mathcal{T}_{3200}$ is dominant (compare with Figure 4).

\section{Cartography}

The goal of this section is to describe some dynamical features of the external resonances by means of the computation of the FLIs, which are suitable chaos indicators allowing us to distinguish between resonant, stable and chaotic motions. In Section 6 we will pay special attention to objects with large area-to-mass ratios.

5.1. Fast Lyapunov Indicators. Making reference to [7], we define the FLI, which corresponds to the largest Lyapunov characteristic exponent at a fixed time, say $t=T$. Precisely, consider the $n$-dimensional differential system

$$
\dot{\mathbf{x}}=\mathbf{f}(\mathbf{x})
$$

where $\mathbf{x} \in \mathbb{R}^{n}$. We write the variational equations as

$$
\frac{d}{d t} \mathbf{v}=\left(\frac{\partial \mathbf{f}(\mathbf{x})}{\partial \mathbf{x}}\right) \mathbf{v}
$$


where $\mathbf{x}$ is an $n$-dimensional vector. For the initial conditions $\mathbf{x}(0) \in \mathbb{R}^{n}, \mathbf{v}(0) \in \mathbb{R}^{n}$ and a time $T \geq 0$, the FLI is defined as

$$
\operatorname{FLI}(\mathbf{x}(0), \mathbf{v}(0), T) \equiv \sup _{0<t \leq T} \log \|\mathbf{v}(t)\| .
$$

We will present different results based on the FLIs, computed in the plane of coordinates or in the parameter plane; the value of the FLI will be marked by a color scale, where darker colors represent a regular dynamics (either periodic or quasi-periodic), while lighter colors are associated to chaotic motions.

5.2. Cartography of the 1:2 resonance. For the 1:2 resonance, we have twelve terms defining the resonant part $R_{\text {earth }}^{\text {res } 1: 2}$ of the geopotential (see Table 2).

Except for eccentricities less than about 0.02 (see Figure 1, top left), the term $\mathcal{T}_{2202}$ is dominant in the rest of the $e-i$ domain. For moderate and large eccentricities, its magnitude is much larger that the size of any other term. As a consequence, it is natural to expect that pendulum like plots are obtained in the $\left(\sigma_{12}, a\right)$ plane (see Figure 2, top panels). The stable equilibrium point is located at $\sigma_{12}=\lambda_{22}+\omega \cong 75^{\circ}+\omega$. For large inclinations and small eccentricities the term $\mathcal{T}_{4200}$ is dominant and the equilibrium is located at $\sigma_{12}=\left(2 \lambda_{42}-180^{\circ}\right) / 2 \cong 31^{\circ}$ (modulus $\left.180^{\circ}\right)$.

Figure 2, middle panels, plots the FLI values as a function of inclination and semimajor axis in order to evaluate the width of the resonance for each value of the inclination and to give a hint on the dynamics inside the resonance. For a specific inclination, the amplitude can be determined by measuring the distance between the two points on the separatrix obtained as the intersection of the vertical line corresponding to that specific inclination and the branches visible on the plot. For small eccentricities (Figure 2, middle left), the amplitude of the resonance is very small (at most two kilometers) in comparison with the amplitude for moderate and high eccentricities (compare with Figure 2, middle right).

The bottom panels of Figure 2 show the FLI values as a function of eccentricity and semimajor axis for small and large inclinations, thus providing an estimate of the width of the resonance in terms of the eccentricity for a fixed value of the inclination.

From these graphs, it follows that the amplitude of the resonance grows in magnitude with the increase of the eccentricity and it reduces in magnitude with the increase of the inclination (see Figure 2, bottom and middle panels).

5.3. Cartography of the 1:3 resonance. A similar analysis can be performed for the 1:3 resonance, where we have several terms defining $R_{\text {earth }}^{\text {res } 1: 3}$ (see Table 2), among which the most important ones are $\mathcal{T}_{3100}$ and $\mathcal{T}_{2204}$ (see Figure 1, top center panel). 

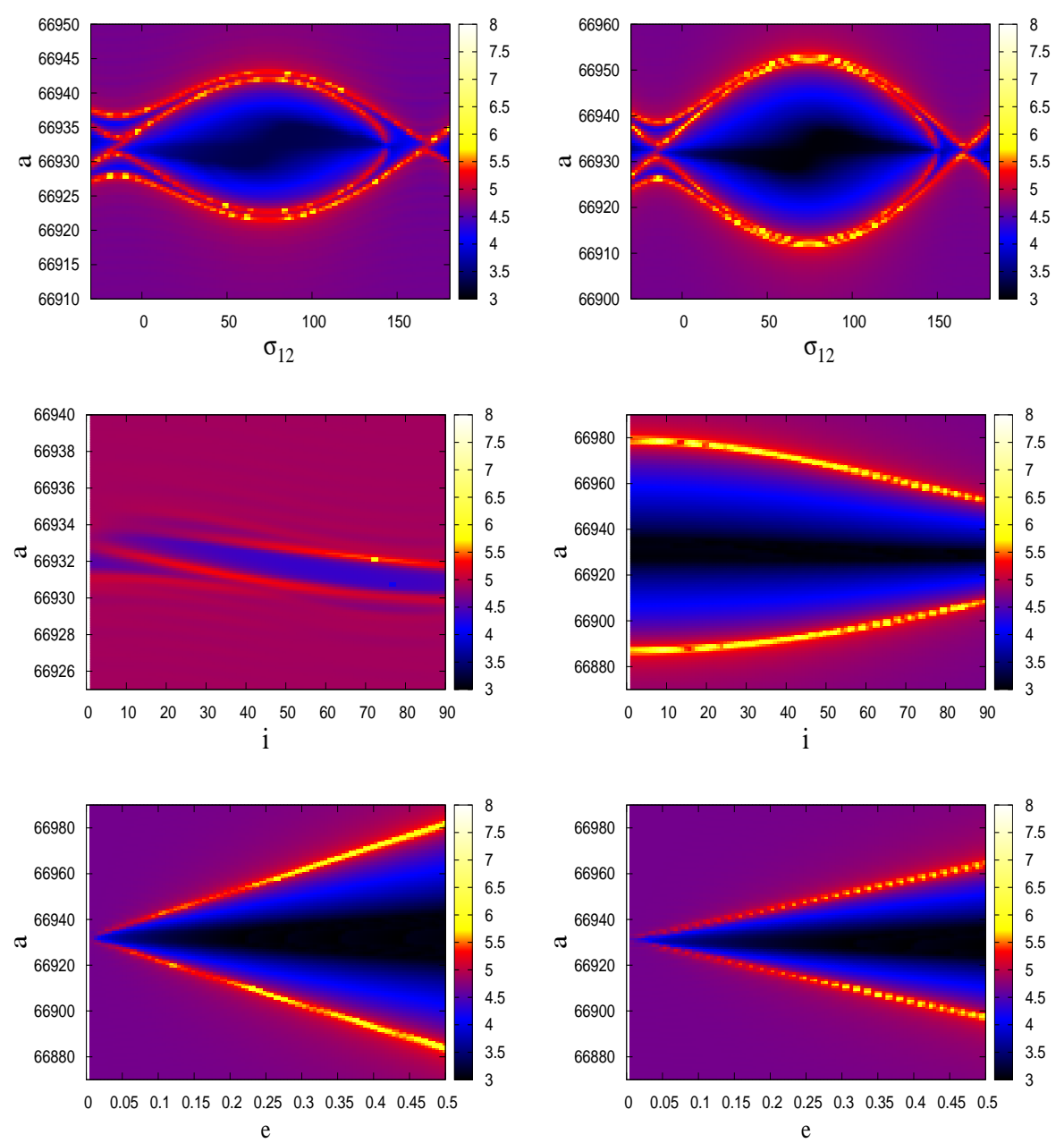

Figure 2. FLI map for the $1: 2$ resonance for $\omega=0^{\circ}, \Omega=0^{\circ}$. Top panels: $e=0.1, i=10^{\circ}$ (left), $e=0.2, i=10^{\circ}$ (right). Middle panels: FLI in the $(i, a)$ plane for $e=0.005, \sigma_{12}=31^{\circ}$ (left), $e=0.5, \sigma_{12}=75^{\circ}$ (right). Bottom panels: FLI in the $(e, a)$ plane for $\sigma_{12}=75^{\circ}$ and $i=20^{\circ}$ (left), $i=70^{\circ}$ (right).

Since $\mathcal{T}_{3100}$ is of order $\mathcal{O}(1)$ (while the other terms are $\mathcal{O}(e)$ ), for small eccentricities some pendulum like plots are obtained, provided the inclination is not zero. The stable equilibrium point is located at $\sigma_{13}=\lambda_{31}-180^{\circ} \cong 7^{\circ}$ (see Figure 3, top left).

On the other hand, since $\mathcal{T}_{2204}$ (which contains $\cos \left(2\left(\sigma_{13}-2 \omega-\lambda_{22}\right)\right)$ ) is dominant for large eccentricities, one gets two stable points, located at $\sigma_{13}=\lambda_{22} \cong 75^{\circ}$ and $\sigma_{13}=\lambda_{22}-180^{\circ} \cong-105^{\circ}$ (see Figure 3 , top right). Notice that, although the term 

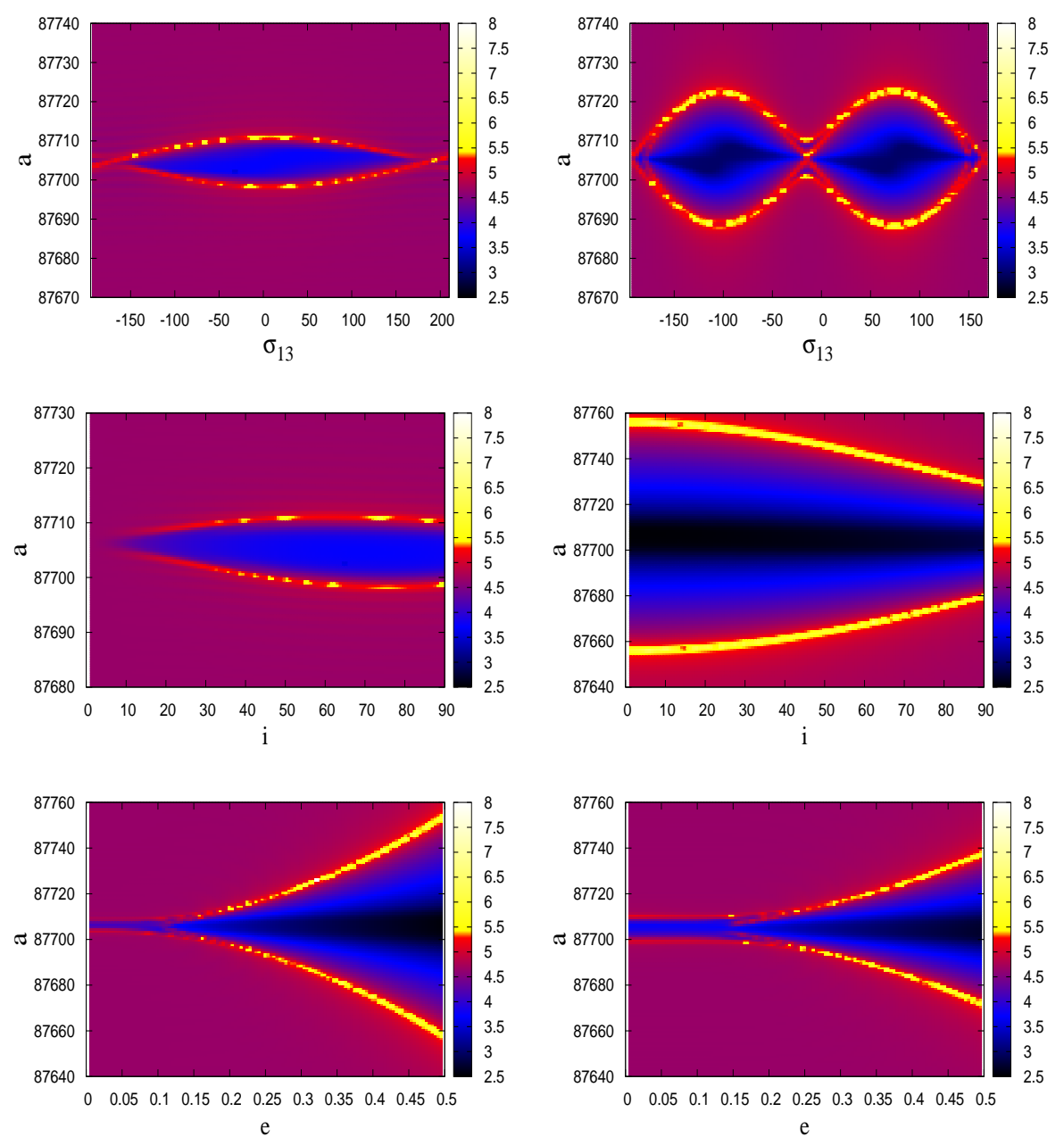

Figure 3. FLI for the $1: 3$ resonance for $\omega=0^{\circ}, \Omega=0^{\circ}$. Top panels: $e=0.005, i=70^{\circ}$ (left); $e=0.3, i=25^{\circ}$ (right). Middle panels: FLI in the $(i, a)$ plane for $e=0.005, \sigma_{13}=7^{\circ}$ (left), $e=0.5, \sigma_{13}=75^{\circ}$ (right). Bottom panels: FLI in the $(e, a)$ plane for $\sigma_{13}=75^{\circ}$ and $i=20^{\circ}$ (left), $i=70^{\circ}$ (right).

$\mathcal{T}_{2204}$ contains a fourth power of the eccentricity, the width of the resonance is larger in comparison with that associated to $\mathcal{T}_{3100}$.

The middle panels of Figure 3 show the FLI values as a function of inclination and semimajor axis, while the bottom panels refer to the $(e, a)$ plane.

5.4. Cartography of the 2:3 resonance. For the $2: 3$ resonance, we have three terms defining $R_{\text {earth }}^{\text {res } 2: 3}$ (see Table 2 ): $\mathcal{T}_{2201}, \mathcal{T}_{3200}$ and $\mathcal{T}_{3212}$. The harmonic term $\mathcal{T}_{3200}$ is dominant 

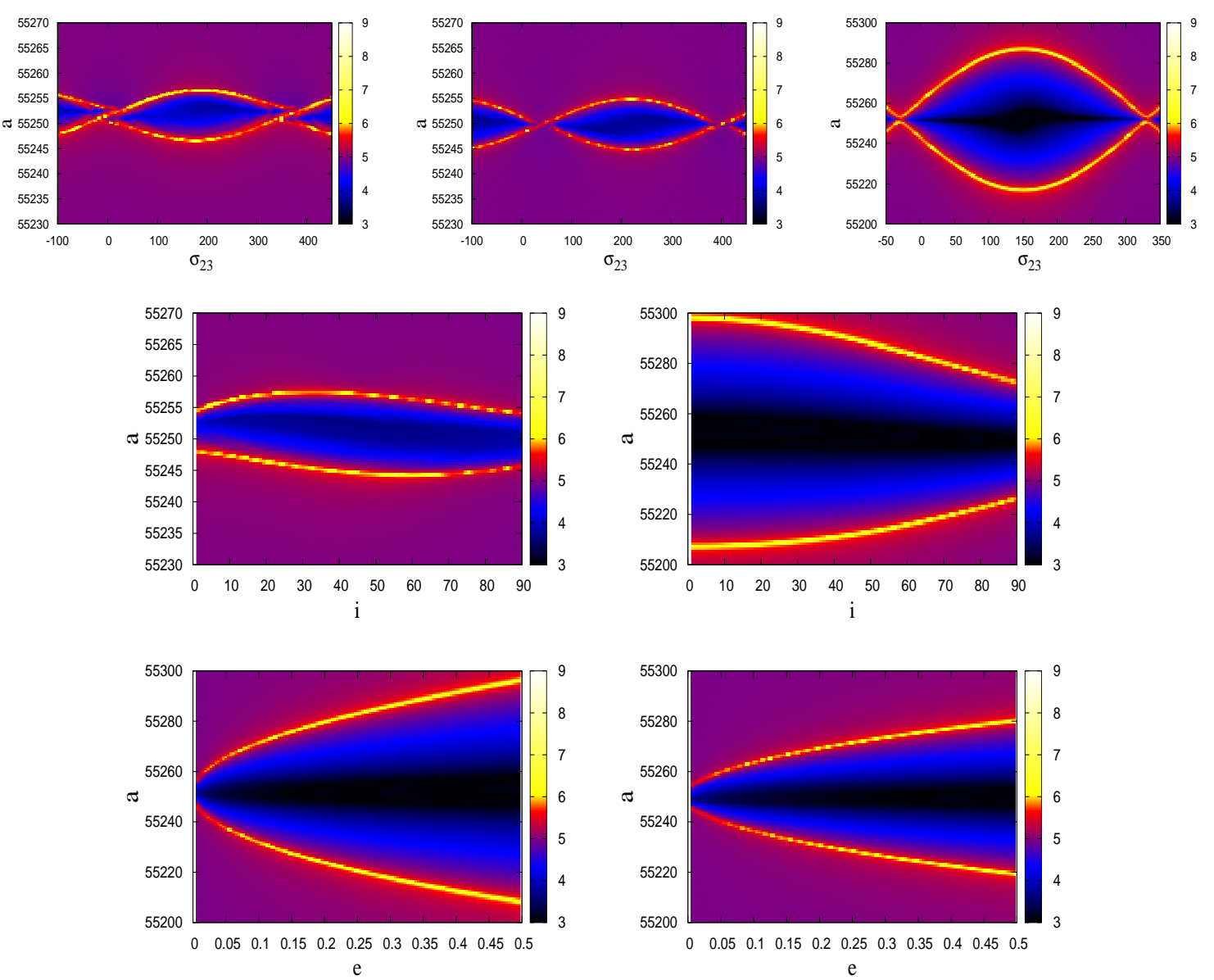

Figure 4. FLI for the $2: 3$ resonance for $\omega=0^{\circ}, \Omega=0^{\circ}$. Top panels: $e=0.005, i=10^{\circ}$ (left); $e=0.005, i=70^{\circ}$ (middle) $e=0.3, i=10^{\circ}$ (right). Middle panels: FLI in the $(i, a)$ plane for $e=0.005, \sigma_{23}=236^{\circ}$ (left), $e=0.5, \sigma_{23}=150^{\circ}$ (right). Bottom panels: FLI in the $(e, a)$ plane for $\sigma_{23}=150^{\circ}$ and $i=20^{\circ}$ (left), $i=70^{\circ}$ (right).

for small eccentricities and nonzero inclinations, while $\mathcal{T}_{2201}$ is dominant for the rest of the domain. Pendulum like plots are obtained for any value of eccentricity and inclination. For small eccentricities the stable equilibrium point is close to $\sigma_{23}=2 \lambda_{22}+\omega \cong 150^{\circ}+\omega$, when the inclination is small (see Figure 4 top left), while it is located in the vicinity of $\sigma_{23}=2 \lambda_{32}+90^{\circ} \cong 236^{\circ}$ when the inclination is large (see Figure 4 top middle). For moderate and high eccentricities, the stable point is located at $\sigma_{23}=2 \lambda_{22}+\omega \cong 150^{\circ}+\omega$ (Figure 4 top right). 
The amplitude of the resonant islands for small and large eccentricities can be deduced from Figure 4, middle panels, while the bottom plots provide the FLI as a function of eccentricity and semimajor axis for (relatively) small and large inclinations.

5.5. A comparison with the full Cartesian equations. The purpose of this section is to complement the study performed by using the Hamiltonian formulation with some results obtained in Cartesian coordinates. In fact, the Hamiltonian formulation provides an approximation of the dynamics, due to the fact that we just consider the secular and resonant expansions. Moreover, these expansions have been developed up to second order in eccentricity. Clearly, the Cartesian equations of motion contain the non-truncated terms of the geopotential, including the short periodic terms, as well as the gravitational attraction of the Sun, Moon and the effect of the solar radiation pressure (see (6.1) below).

Henceforth, the Cartesian formulation allows one to have a more complete model, although at the expense of a much higher computational time, at least with respect to the Hamiltonian formulation. However, the Cartesian approach cannot provide a global explanation of the resonant dynamics as a function of eccentricity and inclination, as it was done in the previous sections using the Hamiltonian formalism. The goal of this section will be to validate the results obtained by means of the resonant Hamiltonian describing an approximation of the geopotential (see Sections 5.2, 5.3, 5.4) by comparing such results with a more complete model, obtained using the Cartesian formalism and including all major effects.

Let us remark that within the Hamiltonian formulation, we removed the short periodic perturbations by averaging over the fast angles, thus leading us to compute the mean orbital elements. As a consequence, when dealing with the equations of motion in Cartesian coordinates, in order to represent the FLI as a function of the same variables as in the Hamiltonian approach, we need to transform from osculating orbital elements to mean elements. This computation implies a numerical average of the osculating elements, which is performed in the course of the integration itself; we refer to [25] for alternative approaches to compare osculating and mean orbital elements, based for example on numerical averaging, fixed-point iterations, or a Hamiltonian transformation.

The results obtained by using the Hamiltonian formulation are validated by integrating the Cartesian equations of motion as shown in Figures 5. Moreover, we evaluate the effects of the other perturbing forces, in particular the influence of the Moon. We have used as starter a single step method (a Butcher numerical algorithm), while a multistep 
predictor-corrector numerical method (Adams-Bashforth 12 steps and Adams-Moulton 11 steps) performs most of the propagation. For each resonance, the total time span was 20000 sidereal days. For each orbit we used a fixed initial tangent vector; a different choice of the tangent vector might alter the results ([7], [15]), although it is not really relevant in our context and, indeed, we leave the exploration of the effect of a random choice of this vector to a future work.

Comparing the above plots with those obtained using the Hamiltonian approach, we are led to the following conclusions: the main dynamical features of the 1:2, 2:3 and 1:3 resonances, namely the location of the equilibrium points and the amplitude of resonant islands, which we already found by using the Hamiltonian formalism, are confirmed by integrating the full equations of motions. However, the other disturbing functions, in particular the perturbations due to the Moon, induce in some cases a substantial effect.

In fact, from the plots of Figure 5 we notice that within the present range of eccentricities below 0.5 the influence of the Moon becomes larger for the orbits located farther from the Earth. For example, for the 1:2 resonance, only small variations are observed when adding the effects of Sun, Moon and SRP (see Figure 5, top left and middle panels) with a good agreement with the Hamiltonian formulation (compare with Figure 2, top right panel). Also the agreement with the 2:3 resonance is very satisfactory (compare Figure 5, top right with Figure 4, top right). On the contrary, in the case of the 1:3 resonance, which is closer to the Moon, the Moon's perturbation leads to large chaotic regions (see Figure 5, bottom panels and compare Figure 3, top right with Figure 5, bottom right). We will see in Section 7 that for large eccentricities the effect of the Moon is more important for the 1:2 resonance, than for the 1:3 resonance. The lunar interaction with the external resonances, as the orbital parameters (i.e., eccentricity and inclination) are varied, will be the subject of a further investigation.

\section{Dynamics of LARGE AREA-TO-MASs RATio OBJECTS}

Extended analyses of the dynamics of high area-to-mass ratio objects are provided in several papers (see, e.g., [4], [16], [17], [21], [22], [23]). The purpose of this section is to explore the interaction of such objects with the 1:2, 2:3, 1:3 resonances, by using the Hamiltonian formalism. For simplicity, we focus our attention on planar orbits, for which we compute the FLI for various values of the area-to-mass parameter. To this end, we proceed to expand the disturbing function corresponding to SRP in terms of the orbital elements. Precisely, the potential describing the effect of SRP, which should be added to 

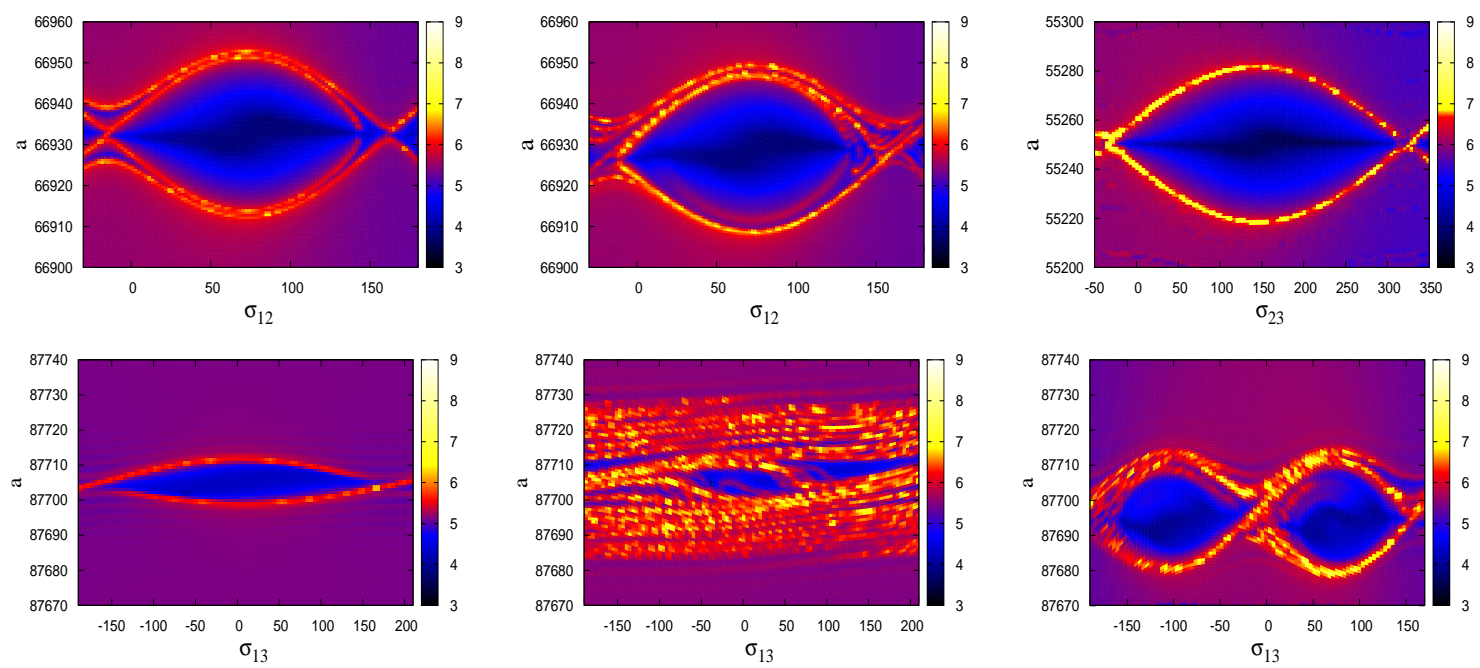

Figure 5. FLI using Cartesian equations. The 1:2 resonance for $e=0.2$, $i=10^{\circ}, \omega=0^{\circ}, \Omega=0^{\circ}$, under the effects of Earth's oblateness (top left), adding Moon + Sun $+\mathrm{SRP}$ with $A / m=0.01\left[\mathrm{~m}^{2} / \mathrm{kg}\right]$ (top middle). The 2:3 resonance for $e=0.3, i=10^{\circ}, \omega=0^{\circ}, \Omega=0^{\circ}$, under the effects of Earth's oblateness + Moon + Sun + SRP with $A / m=0.01\left[m^{2} / k g\right]$ (top right). FLI for the 1:3 resonance with $e=0.005, i=70^{\circ}, \omega=0^{\circ}, \Omega=0^{\circ}$, under the effects of Earth's oblateness (bottom left), adding Moon + Sun $+\mathrm{SRP}$ with $A / m=0.01\left[\mathrm{~m}^{2} / \mathrm{kg}\right]$ (bottom middle), $e=0.3, i=25^{\circ}$, $\omega=0^{\circ}, \Omega=0^{\circ}$, under the effects of Earth's oblateness + Moon + Sun + $\mathrm{SRP}$ with $A / m=0.01\left[\mathrm{~m}^{2} / \mathrm{kg}\right]$ (bottom right).

the Hamiltonian (2.2), can be written as (compare with (2.1))

$$
V_{s r p}=-C_{r} P_{r} a_{S}^{2}\left(\frac{A}{m}\right) \frac{1}{\left|\mathbf{r}-\mathbf{r}_{S}\right|},
$$

where we can use the expansion in terms of the Legendre polynomials:

$$
\frac{1}{\left|\mathbf{r}-\mathbf{r}_{S}\right|}=\frac{1}{r_{S}} \sum_{j=1}^{\infty}\left(\frac{r}{r_{S}}\right)^{j} P_{j}(\cos Q)
$$

with $Q$ being the angle between the Sun and the geocentric radius of the debris (the zeroth order term $\frac{1}{r_{S}}$ in the expansion can be neglected, since it does not contribute to the Hamiltonian). As for the position of the Sun, we use the following formulae borrowed from [18], and normalized with respect to the geostationary distance $a_{\text {geo }}=42164.1696 \mathrm{~km}$ as well as with a unit of time $\tau$ chosen such that the period of Earth's rotation becomes 
equal to $2 \pi$ :

$$
\begin{aligned}
r_{S} & =23.71681950544094\left(149.619-2.499 \cos M_{S}-0.021 \cos 2 M_{S}\right) \\
M_{S} & =\frac{2 \pi}{360}(357.5256+35999.04944 t) \\
\lambda_{S} & =\frac{2 \pi}{360} 282.94+M_{S}+\frac{2 \pi}{360}\left(\frac{6892}{3600} \sin M_{S}+\frac{72}{3600} \sin 2 M_{S}\right) \\
t & =\frac{365.242196 \theta}{36525 \cdot 366.242196 \cdot 2 \pi},
\end{aligned}
$$

where $M_{S}$ is the Sun's mean anomaly and $\lambda_{S}$ is the ecliptic longitude. Casting together these formulae, by the algebraic manipulator Mathematica ${ }^{\circledR}$ we compute the expansion of (6.1) up to the third order of the Legendre polynomials, taking care of neglecting in the expansion those terms which are multiplied by coefficients less than a specific error, whose dimension is $\mathrm{kg} / \mathrm{m}^{2} \mathrm{~d} / \tau^{2}$, and that we fix (after trials and errors) equal to $10^{-9}$. Finally, we average over the mean anomaly to obtain the following expression $V_{s r p}^{a p}$ for the approximate expansion of the potential describing the SRP:

$$
\begin{aligned}
V_{\text {srp }}^{\text {app }} & =a e \frac{A}{m}\left(-4.83810^{-7} \sin (-0.00546061 \theta+\omega)\right. \\
& -4.83610^{-7} \sin (-0.00546061 \theta+\omega)-0.000028751 \sin (-0.0027303 \theta+\omega) \\
& +1.23910^{-6} \sin (0.0027303 \theta+\omega)+5.42510^{-6} \cos (-0.0027303 \theta+\omega) \\
& \left.+1.14110^{-7} \cos (\omega+0.0027303 \theta)\right) .
\end{aligned}
$$

Clearly, the dimension of $V_{s r p}^{a p p}$ is $d^{2} / \tau^{2}$.

In Figures 6, 7, 8, the FLI is computed as a function of the resonant angle and semimajor axis, respectively for the 1:2, 1:3, 2:3 resonances. The canonical equations include the geopotential and the perturbing effect of the solar radiation pressure. The patterns are obtained by using the following area-to-mass ratios: $A / m=0,1,5,15\left[\mathrm{~m}^{2} / \mathrm{kg}\right]$, and are represented in each figure, respectively, in the top left, top right, bottom left and bottom right panels.

By analyzing these results, the main conclusion is the following. Even if the amplitude of the resonance is of the order of a few tens of kilometers (top left plots), the solar radiation pressure effect for objects with high area-to-mass ratios yields a web of secondary resonances which covers an area of several hundred kilometers. For example, in the case of the 1:3 resonance, the amplitude of the resonance for objects with small area-to-mass ratio located in planar eccentric orbits with $e=0.3$ is about 30 kilometers (Figure 7 top left). For $A / m=15\left[\mathrm{~m}^{2} / \mathrm{kg}\right]$ the web of resonances extends on a region of more than 500 kilometers (Figure 7 bottom right). 

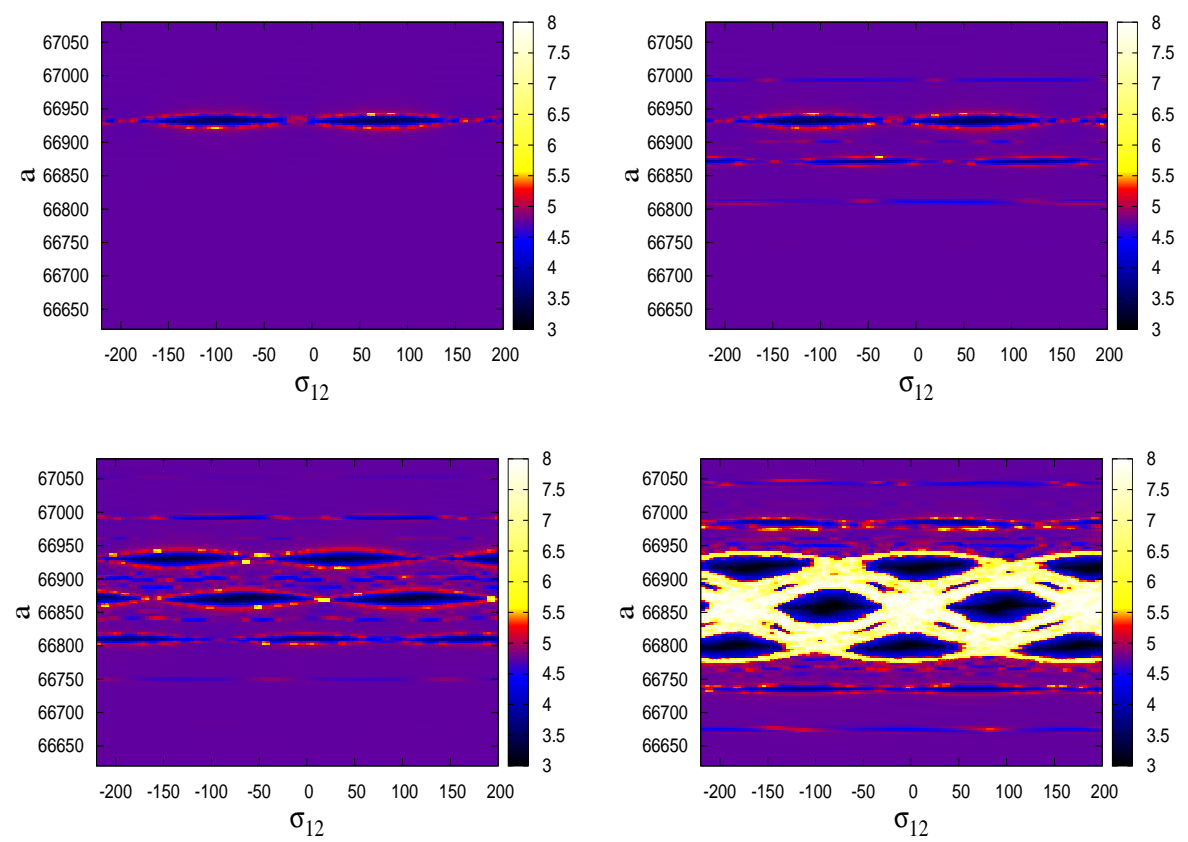

FiguRE 6. FLI for the 1:2 resonance, under the effects of the geopotential and SRP, for $i=0^{\circ}, e=0.1, \omega=0^{\circ}, \Omega=0^{\circ}$ : $A / m=0\left[\mathrm{~m}^{2} / \mathrm{kg}\right]$ (top left); $A / m=1\left[\mathrm{~m}^{2} / \mathrm{kg}\right]$ (top right); $A / m=5\left[\mathrm{~m}^{2} / \mathrm{kg}\right]$ (bottom left); $A / m=15\left[\mathrm{~m}^{2} / \mathrm{kg}\right]$ (bottom right).

\section{Two CASE Studies: XMM-Newton And Integral}

As we mentioned in Section 1, two space missions are directly connected with external resonances. Precisely, XMM-Newton has a semimajor axis located at the 1:2 resonance, while the semimajor axis of Integral is at the 1:3 resonance. However, we must extend the analysis performed in the previous sections, since the orbital eccentricities of such missions are quite large: XMM-Newton has an eccentricity equal to $e=0.776$, while it is $e=0.824$ in the case of Integral. Such high eccentricities lead to consider some different terms in the expansion of the resonant parts associated to these two resonances. Moreover, the functions $G_{n p q}$ will be developed up to a larger order in eccentricity $\left(14^{t h}\right.$ order in the forthcoming computations). In particular, we take $N=3$ and among all harmonic terms up to degree and order $n=m=3$, listed in Table 4 , we consider in the computations just the most important ones. These terms are reported in bold and their explicit expressions are given in Appendix A. 

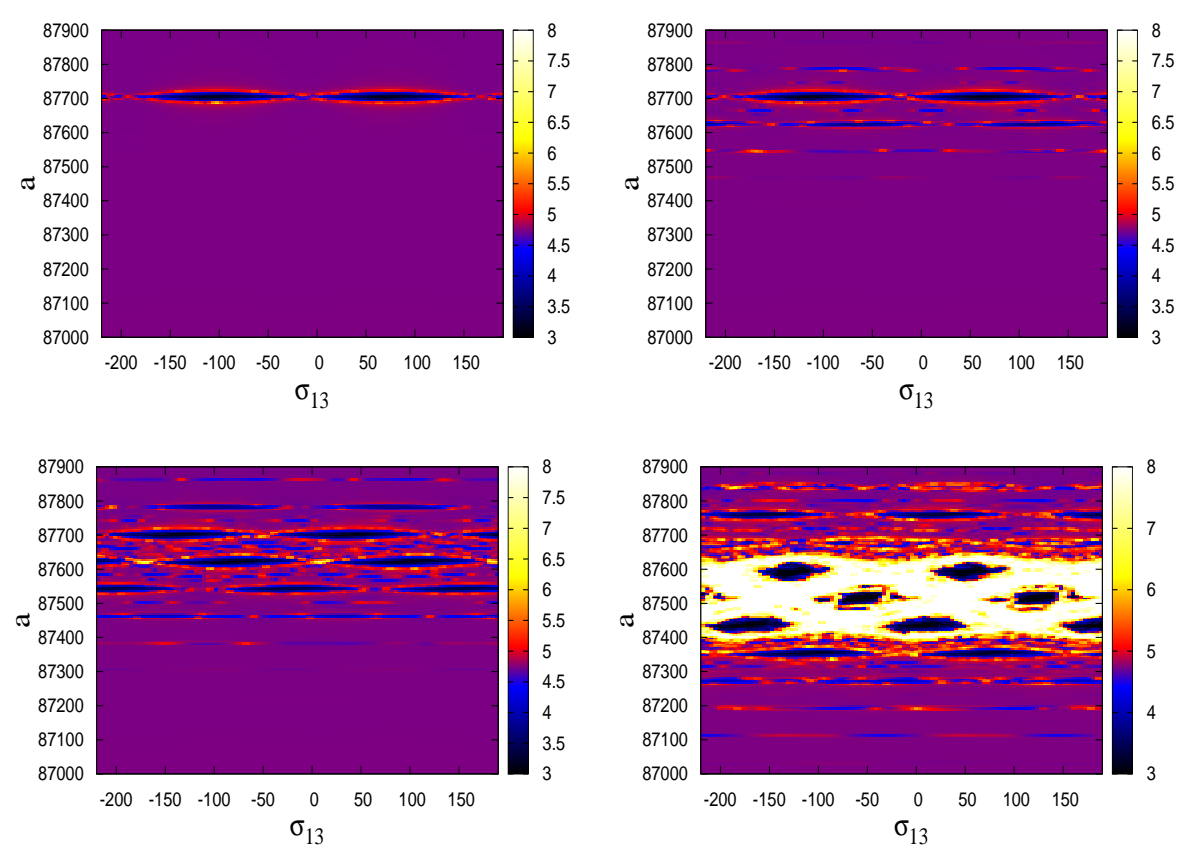

FiguRE 7. FLI for the 1:3 resonance, under the effects of the geopotential and SRP, for $i=0^{\circ}, e=0.3, \omega=0^{\circ}, \Omega=0^{\circ}$ : $A / m=0\left[\mathrm{~m}^{2} / \mathrm{kg}\right]$ (top left); $A / m=1\left[\mathrm{~m}^{2} / \mathrm{kg}\right]$ (top right); $A / m=5\left[\mathrm{~m}^{2} / \mathrm{kg}\right]$ (bottom left); $A / m=15\left[\mathrm{~m}^{2} / \mathrm{kg}\right]$ (bottom right).

\begin{tabular}{|c|c|c|}
\hline$j: \ell$ & $N$ & terms \\
\hline $1: 2$ & 4 & $\mathcal{T}_{\mathbf{2 2 0 2}}, \mathcal{T}_{\mathbf{2 2 1 4}}, \mathcal{T}_{\mathbf{2 2 2 6}}, \mathcal{T}_{\mathbf{3 1 0}-\mathbf{1}}, \mathcal{T}_{\mathbf{3 1 1 1}}, \mathcal{T}_{\mathbf{3 1 2 3}}, \mathcal{T}_{\mathbf{3 2 0 1}}, \mathcal{T}_{\mathbf{3 2 1 3}}, \mathcal{T}_{\mathbf{3 3 0 3}}$, \\
& & $\mathcal{T}_{2100}, \mathcal{T}_{2112}, \mathcal{T}_{2124}, \mathcal{T}_{3135}, \mathcal{T}_{3225}, \mathcal{T}_{3237}, \mathcal{T}_{3315}, \mathcal{T}_{3327}, \mathcal{T}_{3339}$ \\
\hline $1: 3$ & 4 & $\mathcal{T}_{\mathbf{2 2 0 4}}, \mathcal{T}_{\mathbf{2 2 1 6}}, \mathcal{T}_{\mathbf{2 2 2 8}}, \mathcal{T}_{\mathbf{3 1 0 0}}, \mathcal{T}_{\mathbf{3 1 1 2}}$ \\
& & $\mathcal{T}_{2101}, \mathcal{T}_{2113}, \mathcal{T}_{2125}, \mathcal{T}_{3124}, \mathcal{T}_{3136}, \mathcal{T}_{3203}, \mathcal{T}_{3215}, \mathcal{T}_{3227}, \mathcal{T}_{3239}, \mathcal{T}_{3306}, \mathcal{T}_{3318}, \mathcal{T}_{33210}, \mathcal{T}_{33312}$ \\
\hline
\end{tabular}

TABLE 4. Terms for the 1:2 and 1:3 resonances in the case of large eccentricities; the sum of these terms provides $R_{\text {earth }}^{r e s j: \ell}$. The most relevant ones, which are considered in our computations and whose explicit expressions are given in Appendix A, are reported in bold.

The dominant terms for large eccentricities and the amplitudes of the resonances are shown in Figure 9 for the 1:2 and 1:3 resonances. For a fast identification, we also mark with bullets the positions of XMM-Newton (within the plots referring to the 1:2 resonance) and Integral (within those referring to the 1:3 resonance).

Concerning the secular part, since $\left|J_{30}\right| \ll J_{20}$ we neglect all terms of order $J_{30}$. 

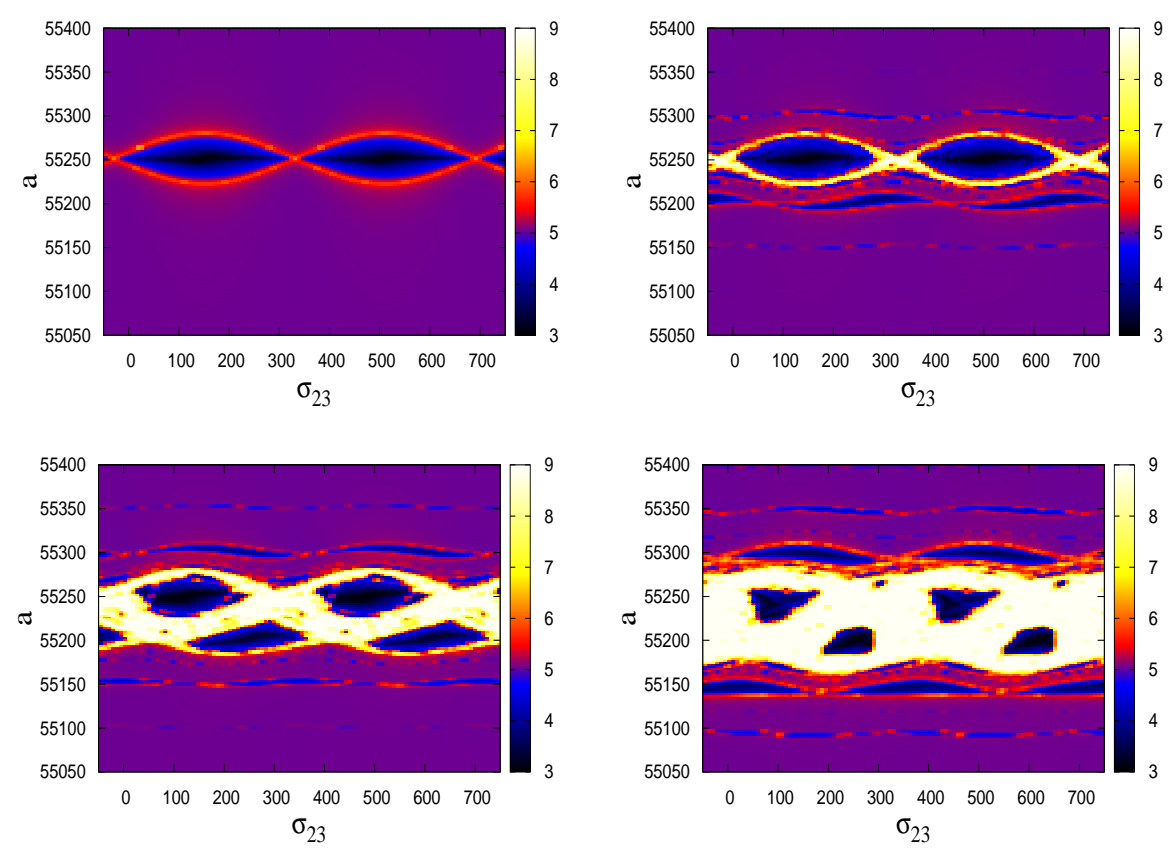

FiguRE 8. FLI for the 2:3 resonance, under the effects of the geopotential and SRP, for $i=0^{\circ}, e=0.2, \omega=0^{\circ}, \Omega=0^{\circ}$ : $A / m=0\left[\mathrm{~m}^{2} / \mathrm{kg}\right]$ (top left); $A / m=1\left[\mathrm{~m}^{2} / \mathrm{kg}\right]$ (top right); $A / m=5\left[\mathrm{~m}^{2} / \mathrm{kg}\right]$ (bottom left); $A / m=15\left[\mathrm{~m}^{2} / \mathrm{kg}\right]$ (bottom right).

Let us first analyze XMM-Newton, whose parameters are $e=0.776$ and $i=65.4^{\circ}([26])$. From Figure 9 we see that the leading term is $\mathcal{T}_{2214}$ with an amplitude of the resonance around $60-70 \mathrm{~km}$. The orbital data of Integral correspond to $e=0.824$ and $i=52.2^{\circ}$ ([13]). In this case $\mathcal{T}_{2216}$ is the dominant term with an amplitude of the resonance of about $70 \mathrm{~km}$. These values are in agreement with the FLI plots shown in Figure 10.

The stable equilibrium point for the $1: 2$ resonance corresponds to the value of $\sigma_{12}=$ $2 \omega+\lambda_{22}\left(\operatorname{modulus} 180^{\circ}\right.$ ) with $\omega=93^{\circ}$ and $\lambda_{22}=75^{\circ}$; therefore, the equilibrium is located at $81^{\circ}$ as shown in the upper left panel of Figure 10. Due to the interplay between the terms associated to $J_{22}$ and those to $J_{31}, J_{32}, J_{33}$, we have a peculiar pattern with a double shape surrounding the main island.

As for the 1:3 resonance, the location of the equilibrium points is influenced by both the white and black terms of Figure 9. More precisely, since $\omega=302^{\circ}, \lambda_{22}=75^{\circ}$ the dominant white term gives rise to an equilibrium point at $\sigma_{13}=3 \omega+\lambda_{22}$ (modulus $180^{\circ}$ ) $\simeq 261^{\circ}$, while the equilibrium point corresponding to the black term is at $\sigma_{13}=2 \omega+\lambda_{22}$ 

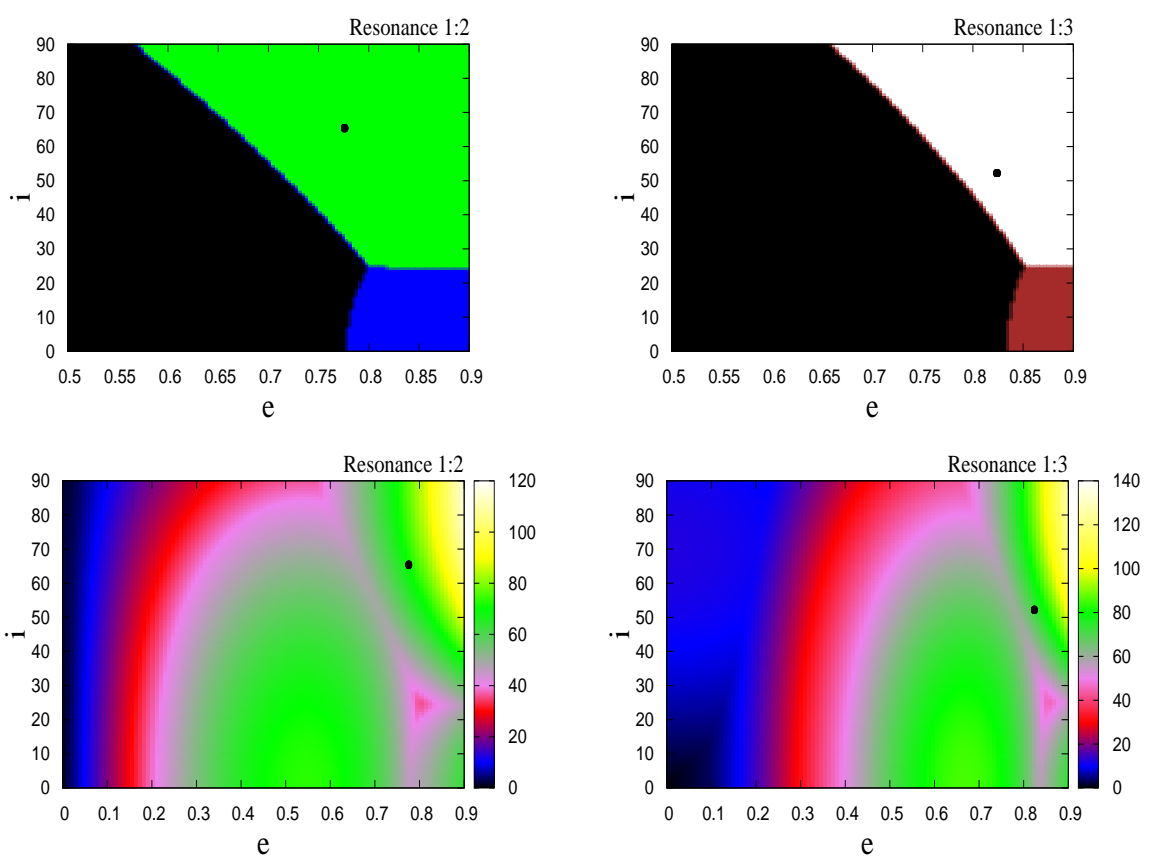

FiguRE 9. Dominant terms (upper panels) and amplitudes (lower panels) of the 1:2 (left panels) and 1:3 (right panels) resonances. The black dots represent XMM-Newton on the left panels and Integral in the right panels. The colors of the dominant terms are the following. For the 1:2 resonance: $\mathcal{T}_{2202}$ black, $\mathcal{T}_{3111}$ blue, $\mathcal{T}_{2214}$ green; for the 1:3 resonance: $\mathcal{T}_{2204}$ black, $\mathcal{T}_{3112}$ brown, $\mathcal{T}_{2216}$ white. The color bar in the lower panels provides the amplitude of the resonance in kilometers.

(modulus $\left.180^{\circ}\right) \simeq 319^{\circ}$. Because these two terms are comparable in magnitude, the stable equilibrium point is in fact located between $261^{\circ}$ and $316^{\circ}$, closer to $261^{\circ}$, as shown in the upper right panel of Figure 10.

The middle panels of Figure 10 show the integration of the Cartesian equations for 8000 sidereal days, including the effects of the Sun, the Moon and the solar radiation pressure. The comparison between the Hamiltonian and Cartesian plots (upper and middle panels in Figure 10) is quite different for the 1:2 and 1:3 resonance. Indeed, for the 1:3 resonance we have that the Hamiltonian plot provides a fairly exact location of the stable equilibrium as well as a good approximation of the separatrix. Of course, the dynamics within the resonance island is affected by the influence of Sun, Moon, SRP as well as all other terms providing the geopotential at such high eccentricities. For 

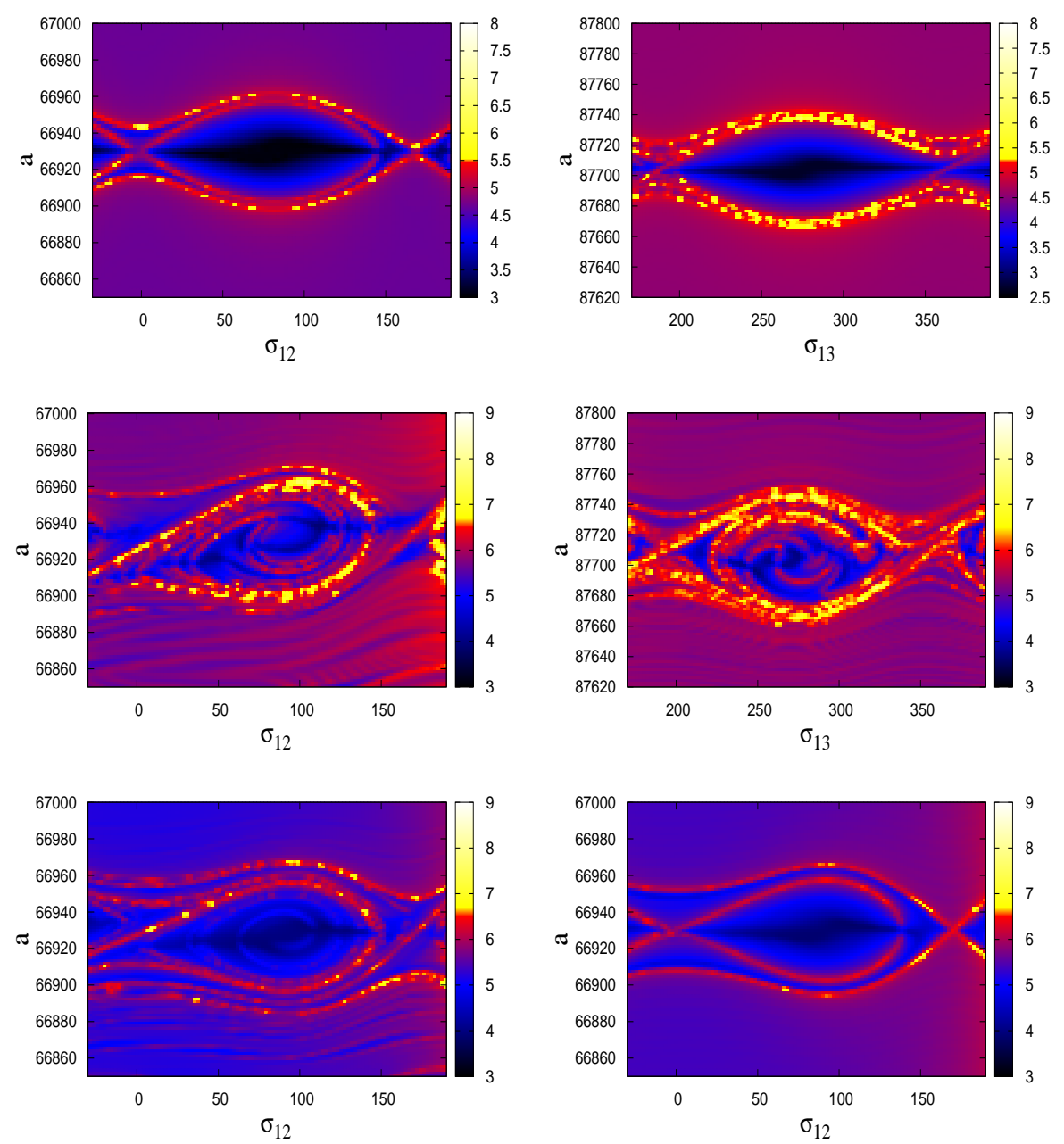

Figure 10. Top left: FLI for the 1:2 resonance for $e=0.776, i=65.4^{\circ}$, $\omega=93.3^{\circ}, \Omega=55.5^{\circ}$. Top right: FLI for the $1: 3$ resonance for $e=0.824$, $i=52.2^{\circ}, \omega=302^{\circ}, \Omega=103^{\circ}$. Middle panels: FLI in Cartesian formalism for the 1:2 (left) and 1:3 resonance (right). Bottom panels: FLI in Cartesian formalism for the 1:2 resonance without the effect of the Moon (left) and without the effect of Moon, Sun, SRP (right).

the 1:2 resonance the results are not comparably good (upper and middle left panels in Figure 10), since we observe a distortion and displacement of the separatrix. To highlight which effect is dominant for the 1:2 resonance, we have integrated the Cartesian equations eliminating the effect of the Moon (bottom left panel in Figure 10) and then eliminating also the effects of the Sun and SRP (bottom right panel in Figure 10). The results show 
that the Moon is definitely important in shaping the dynamics of the 1:2 resonance, while Sun and SRP have secondary, although still important, effects.

\section{Conclusions And PERspeCtives}

The dynamics of satellites and debris in external resonances is well approximated by the Hamiltonian approach based on the study of geopotential including the secular and resonant terms. In particular, elementary mathematical techniques provide a fast and reliable procedure to reduce the study to the dominant terms (instead of considering long series expansions, thus considerably decreasing the computational effort), to locate the equilibrium points and to determine their stability, to measure the amplitude of the resonance (see Section 4). All these information provide us with a strong background, which makes easier to investigate the dynamics of real bodies, when additional effects, like those of Sun, Moon and SRP, are considered.

It is worth to underline that our analysis refers mainly to the long term evolution of the semimajor axis, which is influenced directly by the resonant part of the geopotential. Therefore, we focused especially on this aspect of the dynamics. However, one should mention that the disturbing functions due to Moon, Sun and solar radiation pressure also influence the long term behavior of the semimajor axis, though indirectly. More precisely, their associated Fourier expansions, with respect to the orbital elements, do not contain resonant harmonic terms and, therefore, the canonical equation describing the evolution of the semimajor axis (or $L$ ) do not change its form, when these additional perturbations are considered. However, the long term evolution of the other orbital elements (equivalently, the other Delaunay variables) are directly affected by these perturbations. In particular, the eccentricity and inclination vary slowly over time and therefore they indirectly affect the evolution of the semimajor axis, since the canonical equation describing the evolution of the semimajor axis involves all orbital elements as parameters. The problem is much more complex when shadow effects are considered, especially for high area to mass ratio objects. In conclusion, in order to get a clearer picture of how the semimajor axis evolves in time, as a result of the interaction of all disturbing forces, one needs to quantify the effects of all perturbations as a function of the orbital elements.

The alternative approach consists in the numerical integration of the Cartesian equations of motion. In this case it is straightforward to include the effects of Sun, Moon and SRP, at the expenses of a longer computational time needed to have equivalent plots as in the Hamiltonian case. Moreover, the Cartesian approach, although providing a 
validation of the Hamiltonian results, does not allow to provide information about the location of the equilibria as well as about the shape and width of the resonant region, as it was done instead by implementing the Hamiltonian procedure.

The Hamiltonian approach was also used to investigate the behavior of objects with large area-to-mass ratio by suitably expanding the potential describing the effect of SRP (see Section 6). The results show the appearance of a web of secondary resonances ranging over an area of several hundreds kilometers. In view of these results, it would be interesting to explore the possibility to play with the area-to-mass ratio to stabilize objects within specific resonant islands or rather to move them through the web of resonances and chaotic layers, which appear when $A / m$ is large.

In Section 7 we have considered two specific space missions: XMM-Newton, with a semimajor axis at the 1:2 resonance, and Integral, with a semimajor axis at the 1:3 resonance. In both cases the eccentricity is high and therefore we extended our analysis by including different terms in the series expansion of the geopotential. Although for smaller eccentricities we noticed that the effect of the Moon was more relevant for the 1:3 resonance, in the case of larger eccentricities we noticed that the lunar attraction provokes drastic changes in the shape of both the 1:2 and 1:3 the resonant islands. This result suggests that the rôle of the Moon, as well as that of the Sun - of course, should be carefully analyzed (as it will be done in a later work) as a function of distance, eccentricity and inclination, in order to have an effective and complete description of the dynamics.

\section{Appendix A. LeAding terms}

We report below the leading terms of the expansion of the secular part given in (3.2). For eccentricities larger than 0.5 (Section 7 ), we considered just the effect of $\mathcal{T}_{2010}$ :

$$
\begin{aligned}
& \mathcal{T}_{200-2}=\mathcal{T}_{2022}=0 \\
& \mathcal{T}_{2010}=\frac{\mu_{E} R_{E}^{2} J_{2}}{a^{3}}\left(\frac{3}{4} \sin ^{2} i-\frac{1}{2}\right)\left(1-e^{2}\right)^{-3 / 2} \\
& \mathcal{T}_{301-1}=\mathcal{T}_{3021}=\frac{\mu_{E} R_{E}^{3} J_{3}}{a^{4}}\left(\frac{15}{16} \sin ^{3} i-\frac{3}{4} \sin i\right) e\left(1-e^{2}\right)^{-5 / 2} \sin \omega \\
& \mathcal{T}_{401-2}=\mathcal{T}_{4032}=\frac{\mu_{E} R_{E}^{4} J_{4}}{a^{5}}\left(-\frac{35}{32} \sin ^{4} i+\frac{15}{16} \sin ^{2} i\right) \frac{3 e^{2}}{4}\left(1-e^{2}\right)^{-7 / 2} \cos (2 \omega), \\
& \mathcal{T}_{4020}=\frac{\mu_{E} R_{E}^{4} J_{4}}{a^{5}}\left(\frac{105}{64} \sin ^{4} i-\frac{15}{8} \sin ^{2} i+\frac{3}{8}\right)\left(1+\frac{3 e^{2}}{2}\right)\left(1-e^{2}\right)^{-7 / 2}
\end{aligned}
$$

We report below the terms of the expansion of the 1:2 resonance, listed in Table 2 or written in bold in Table 4. For eccentricities smaller than 0.5, the terms written below 
have been considered up to second order in the eccentricity. For eccentricities larger than 0.5 (Section 7), we considered just the terms for which we wrote the corresponding eccentricity functions $G_{n p q}$ up to order $14^{\text {th }}$ :

$$
\begin{aligned}
& \mathcal{T}_{2100}=\frac{\mu_{E} R_{E}^{2} J_{21}}{a^{3}}\left\{\frac{3}{4} \sin i(1+\cos i)\left(1-\frac{5 e^{2}}{2}\right) \sin \left(\sigma_{12}-\lambda_{21}\right)\right\}, \\
& \mathcal{T}_{2112}=-\frac{\mu_{E} R_{E}^{2} J_{21}}{a^{3}}\left\{\frac{3}{2} \sin i \cos i \frac{9 e^{2}}{4} \sin \left(\sigma_{12}-2 \omega-\lambda_{21}\right)\right\}, \\
& \mathcal{T}_{2202}=\frac{\mu_{E} R_{E}^{2} J_{22}}{a^{3}}\left\{\frac{3}{4}(1+\cos i)^{2} G_{202} \cos \left[2\left(\sigma_{12}-\omega-\lambda_{22}\right)\right]\right\}, \\
& \mathcal{T}_{2214}=\frac{\mu_{E} R_{E}^{2} J_{22}}{a^{3}}\left\{\frac{3}{2}\left(1-\cos ^{2} i\right) G_{214} \cos \left[2\left(\sigma_{12}-2 \omega-\lambda_{22}\right)\right]\right\}, \\
& \mathcal{T}_{2226}=\frac{\mu_{E} R_{E}^{2} J_{22}}{a^{3}}\left\{\frac{3}{4}(1-\cos i)^{2} G_{226} \cos \left[2\left(\sigma_{12}-3 \omega-\lambda_{22}\right)\right]\right\}, \\
& \mathcal{T}_{310-1}=-\frac{\mu_{E} R_{E}^{3} J_{31}}{a^{4}}\left\{\frac{15}{16} \sin ^{2} i(1+\cos i) G_{30-1} \cos \left(\sigma_{12}+\omega-\lambda_{31}\right)\right\}, \\
& \mathcal{T}_{3111}=\frac{\mu_{E} R_{E}^{3} J_{31}}{a^{4}}\left\{\left(\frac{15}{16} \sin ^{2} i(1+3 \cos i)-\frac{3}{4}(1+\cos i)\right) G_{311} \cos \left(\sigma_{12}-\omega-\lambda_{31}\right)\right\}, \\
& \mathcal{T}_{3201}=\frac{\mu_{E} R_{E}^{3} J_{32}}{a^{4}}\left\{\frac{15}{8} \sin i(1+\cos i)^{2} G_{301} \sin \left(2 \sigma_{12}-\omega-2 \lambda_{32}\right)\right\}, \\
& \mathcal{T}_{3123}=\frac{\mu_{E} R_{E}^{3} J_{31}}{a^{4}}\left\{\left(\frac{15}{16} \sin ^{2} i(1-3 \cos i)-\frac{3}{4}(1-\cos i)\right) G_{323} \cos \left(\sigma_{12}-3 \omega-\lambda_{31}\right)\right\}, \\
& \mathcal{T}_{3213}=\frac{\mu_{E} R_{E}^{3} J_{32}}{a^{4}}\left\{\frac{15}{8} \sin i\left(1-2 \cos i-3 \cos ^{2} i\right) G_{313} \sin \left[2\left(\sigma_{12}-2 \omega-\lambda_{32}\right)+\omega\right]\right\}, \\
& \mathcal{T}_{3303}=\frac{\mu_{E} R_{E}^{3} J_{33}}{a^{4}}\left\{\frac{15}{8}\left(1+3 \cos i+3 \cos ^{2} i+\cos ^{3} i\right) G_{303} \cos \left[3\left(\sigma_{12}-\omega-\lambda_{33}\right)\right]\right\}, \\
& \mathcal{T}_{410-2}=\frac{\mu_{E} R_{E}^{4} J_{41}}{a^{5}}\left\{-\frac{35}{32} \sin ^{3} i(1+\cos i) \frac{e^{2}}{2} \sin \left(\sigma_{12}+2 \omega-\lambda_{41}\right)\right\}, \\
& \mathcal{T}_{4110}=\frac{\mu_{E} R_{E}^{4} J_{41}}{a^{5}}\left\{\left(\frac{35}{16} \sin ^{3} i(1+2 \cos i)-\frac{15}{8}(1+\cos i) \sin i\right)\left(1+e^{2}\right) \sin \left(\sigma_{12}-\lambda_{41}\right)\right\}, \\
& \mathcal{T}_{4122}=\frac{\mu_{E} R_{E}^{4} J_{41}}{a^{5}}\left\{\cos i\left(\frac{15}{4} \sin i-\frac{105}{16} \sin ^{3} i\right) 5 e^{2} \sin \left(\sigma_{12}-2 \omega-\lambda_{41}\right)\right\}, \\
& \mathcal{T}_{4200}=\frac{\mu_{E} R_{E}^{4} J_{42}}{a^{5}}\left\{-\frac{105}{32} \sin ^{2} i(1+\cos i)^{2}\left(1-11 e^{2}\right) \cos \left[2\left(\sigma_{12}-\lambda_{42}\right)\right]\right\}, \\
& \mathcal{T}_{4212}=\frac{\mu_{E} R_{E}^{4} J_{42}}{a^{5}}\left\{\left(\frac{105}{8} \sin ^{2} i \cos i(1+\cos i)-\frac{15}{8}(1+\cos i)^{2}\right) \frac{53 e^{2}}{4} \cos \left[2\left(\sigma_{12}-\omega-\lambda_{42}\right)\right]\right\}, \\
& \mathcal{T}_{4302}=\frac{\mu_{E} R_{E}^{4} J_{43}}{a^{5}}\left\{\frac{105}{16} \sin i(1+\cos i)^{3} \frac{51 e^{2}}{2} \sin \left(3 \sigma_{12}-2 \omega-3 \lambda_{43}\right)\right\},
\end{aligned}
$$


where

$$
\begin{aligned}
& G_{202}=\frac{17}{2} e^{2}-19.167 e^{4}+12.521 e^{6}-3.953 e^{8}+0.703 e^{10}-0.114 e^{12}-0.014 e^{14} \\
& G_{214}=4.813 e^{4}+0.806 e^{6}+2.809 e^{8}+2.661 e^{10}+2.941 e^{12}+3.144 e^{14} \\
& G_{226}=0.0889 e^{6}+0.044 e^{8}+0.044 e^{10}+0.036 e^{12}+0.03 e^{14} \\
& G_{30-1}=-e+1.25 e^{3}-0.146 e^{5}+0.08 e^{7}+0.042 e^{9}+0.034 e^{11}+0.027 e^{13} \\
& G_{311}=3 e+2.75 e^{3}+5.104 e^{5}+7.234 e^{7}+9.697 e^{9}+12.403 e^{11}+15.335 e^{13} \\
& G_{301}=5 e-22 e^{3}+25.292 e^{5}-10.889 e^{7}+2.843 e^{9}-0.27 e^{11}+0.151 e^{13} \\
& G_{323}=1.917 e^{3}+3.708 e^{5}+5.899 e^{7}+8.358 e^{9}+11.065 e^{11}+13.997 e^{13} \\
& G_{313}=12.833 e^{3}-0.521 e^{5}+9.898 e^{7}+10.008 e^{9}+13.128 e^{11}+16.009 e^{13} \\
& G_{303}=40.75 e^{3}-161.063 e^{5}+217.8 e^{7}-141.946 e^{9}+55.715 e^{11}-14.141 e^{13}
\end{aligned}
$$

and

$$
\sigma_{12}=2 M-\theta+2 \omega+\Omega
$$

We report below the terms of the expansion of the 1:3 resonance, listed in Table 2 or written in bold in Table 4. For eccentricities smaller than 0.5, the terms written below have been considered up to second order in the eccentricity. For eccentricities larger than 0.5 (Section 7), we considered just the terms for which we wrote explicitly the corresponding eccentricity functions $G_{n p q}$ up to order $14^{\text {th }}$ :

$$
\begin{aligned}
& \mathcal{T}_{2101}=\frac{\mu_{E} R_{E}^{2} J_{21}}{a^{3}}\left\{\frac{3}{4} \sin i(1+\cos i) \frac{7 e}{2} \sin \left(\sigma_{13}-\omega-\lambda_{21}\right)\right\} \\
& \mathcal{T}_{2204}=\frac{\mu_{E} R_{E}^{2} J_{22}}{a^{3}}\left\{\frac{3}{4}(1+\cos i)^{2} G_{204} \cos \left[2\left(\sigma_{13}-2 \omega-\lambda_{22}\right)\right]\right\}, \\
& \mathcal{T}_{2216}=\frac{\mu_{E} R_{E}^{2} J_{22}}{a^{3}}\left\{\frac{3}{2}\left(1-\cos ^{2} i\right) G_{216} \cos \left[2\left(\sigma_{13}-3 \omega-\lambda_{22}\right)\right]\right\}, \\
& \mathcal{T}_{2228}=\frac{\mu_{E} R_{E}^{2} J_{22}}{a^{3}}\left\{\frac{3}{4}(1-\cos i)^{2} G_{228} \cos \left[2\left(\sigma_{13}-4 \omega-\lambda_{22}\right)\right]\right\}, \\
& \mathcal{T}_{3100}=-\frac{\mu_{E} R_{E}^{3} J_{31}}{a^{4}}\left\{\frac{15}{16} \sin ^{2} i(1+\cos i) G_{300} \cos \left(\sigma_{13}-\lambda_{31}\right),\right. \\
& \mathcal{T}_{3112}=\frac{\mu_{E} R_{E}^{3} J_{31}}{a^{4}}\left\{\left(\frac{15}{16} \sin ^{2} i(1+3 \cos i)-\frac{3}{4}(1+\cos i)\right) G_{312} \cos \left(\sigma_{13}-2 \omega-\lambda_{31}\right)\right\}
\end{aligned}
$$




$$
\begin{aligned}
& \mathcal{T}_{410-1}=\frac{\mu_{E} R_{E}^{4} J_{41}}{a^{5}}\left\{\frac{35}{32} \sin ^{3} i(1+\cos i) \frac{3 e}{2} \sin \left(\sigma_{13}+\omega-\lambda_{41}\right)\right\}, \\
& \mathcal{T}_{4111}=\frac{\mu_{E} R_{E}^{4} J_{41}}{a^{5}}\left\{\left(\frac{35}{16} \sin ^{3} i(1+2 \cos i)-\frac{15}{8}(1+\cos i) \sin i\right) \frac{9 e}{2} \sin \left(\sigma_{13}-\omega-\lambda_{41}\right)\right\}, \\
& \mathcal{T}_{4202}=\frac{\mu_{E} R_{E}^{4} J_{42}}{a^{5}}\left\{-\frac{105}{32} \sin ^{2} i(1+\cos i)^{2} \frac{51 e^{2}}{2} \cos \left[2\left(\sigma_{13}-\omega-\lambda_{42}\right)\right]\right\},
\end{aligned}
$$

where

$$
\begin{aligned}
& G_{204}=33.3125 e^{4}-86.4188 e^{6}+81.349 e^{8}-40.741 e^{10}+12.781 e^{12}-2.822 e^{14}, \\
& G_{216}=9.897 e^{6}-3.905 e^{8}+5.445 e^{10}+2.201 e^{12}+3.288 e^{14}, \\
& G_{228}=0.163 e^{8}+0.027 e^{10}+0.067 e^{12}+0.048 e^{14}, \\
& G_{300}=1-6 e^{2}+6.609 e^{4}-1.953 e^{6}+0.46 e^{8}+0.051 e^{10}+0.07 e^{12}+0.055 e^{14}, \\
& G_{312}=6.625 e^{2}+2.437 e^{4}+6.876 e^{6}+8.722 e^{8}+11.365 e^{10}+14.181 e^{12}+17.218 e^{14},
\end{aligned}
$$

and

$$
\sigma_{13}=3 M-\theta+3 \omega+\Omega
$$

We report below the terms of the expansion of the 2:3 resonance, listed in Table 2:

$$
\begin{aligned}
& \mathcal{T}_{2201}=\frac{\mu_{E} R_{E}^{2} J_{22}}{a^{3}}\left\{\frac{3}{4}(1+\cos i)^{2} \frac{7 e}{2} \cos \left(\sigma_{23}-\omega-2 \lambda_{22}\right)\right\}, \\
& \mathcal{T}_{3200}=\frac{\mu_{E} R_{E}^{3} J_{32}}{a^{4}}\left\{\frac{15}{8} \sin i(1+\cos i)^{2}\left(1-6 e^{2}\right) \sin \left(\sigma_{23}-2 \lambda_{32}\right)\right\}, \\
& \mathcal{T}_{3212}=\frac{\mu_{E} R_{E}^{3} J_{32}}{a^{4}}\left\{\frac{15}{8} \sin i\left(1-2 \cos i-3 \cos ^{2} i\right) \frac{53 e^{2}}{8} \sin \left(\sigma_{23}-2 \omega-2 \lambda_{32}\right)\right\},
\end{aligned}
$$

and

$$
\sigma_{23}=3 M-2 \theta+3 \omega+2 \Omega
$$

Acknowledgements. We deeply thank Alessandro Rossi for very useful discussions and suggestions. We are also indebted with the European Network (MC-ITN) Stardust for proposing and sharing stimulating research activities.

\section{ReFERENCES}

[1] A. Celletti, Stability and Chaos in Celestial Mechanics, Springer-Verlag, Berlin; published in association with Praxis Publishing Ltd. (Chichester, ISBN: 978-3-540-85145-5, 2010).

[2] A. Celletti, C. Galeş, On the dynamics of space debris: 1:1 and 2:1 resonances, J. Nonlinear Science, 24, n. 6, 1231-1262, 2014.

[3] C.C. Chao, Applied Orbit Perturbation and Maintenance, Aerospace Press Series, AIAA (Reston, Virgina, 2005).

[4] C. Colombo, C. Lücking, C.R. McInnes, Orbital dynamics of high area-to-mass ratio spacecraft with $J_{2}$ and solar radiation pressure for novel Earth observation and communication services, Acta Astronautica, 81, 137-150, 2012. 
[5] Earth Gravitational Model 2008, http : //earth-info.nga.mil/GandG/wgs84/gravitymod/egm2008/

[6] T.A. Ely, K.C. Howell, Dynamics of articial satellite orbits with tesseral resonances including the effects of luni-solar perturbations, Dynamics and Stability of Systems, 12, n. 4, 243-269, 1997.

[7] C. Froeschlé, E. Lega, R. Gonczi, Fast Lyapunov indicators. Application to asteroidal motion, Celest. Mech. Dyn. Astr., 67, 41-62, 1997.

[8] C. Galeş, A cartographic study of the phase space of the restricted three body problem. Application to the Sun-Jupiter-Asteroid system, Comm. Nonlinear Sc. Num. Sim., 17, 4721-4730, 2012.

[9] G. Gedeon, Tesseral resonance effects on satellite orbits, Cel. Mech., 1, n. 2, 167-189, 1969.

[10] Ch. Hubaux, A. Lemaître, The impact of Earth's shadow on the long-term evolution of space debris, Celest. Mech. Dyn. Astr., 116, 79-95, 2013.

[11] W.M. Kaula, Theory of Satellite Geodesy, Blaisdell Publ. Co. (1966).

[12] H. Klinkrad, Space Debris: Models and Risk Analysis, Springer-Praxis (Berlin-Heidelberg, 2006).

[13] P.L. Jensen, K. Clausen, C. Cassi, F. Ravera, G. Janin, C. Winkler, R. Much, The INTEGRAL spacecraft - in-orbit performance, Astron. Astroph., 411, n. 1, L7-L17, 2003.

[14] M. T. Lane, An analytical treatment of resonance effects on satellite orbits, Cel. Mech., 42, n. 1-4, 3-38, 1987.

[15] E. Lega, C. Froeschlé, On the relationship between Fast Lyapunov Indicator and periodic orbits for symplectic mappings, in "Dynamics of Natural and Artificial Celestial Bodies", H. Pretka-Ziomek, E. Wnuk, P.K. Seidelmann, D.L. Richardson eds., Springer, Netherlands, 2001.

[16] A. Lemaître, N. Delsate, S. Valk, A web of secondary resonances for large $A / m$ geostationary debris, Celest. Mech. Dyn. Astr., 104, 383-402, 2009.

[17] C. Lücking, C. Colombo, C.R. McInnes, A passive satellite deorbiting strategy for medium Earth orbit using solar and $J_{2}$ effect, Acta Astronautica, 77, 197-206, 2012.

[18] O. Montenbruck, E. Gill, Satellite orbits, Springer (2000).

[19] A. Rossi, Resonant dynamics of Medium Earth Orbits: space debris issues, Celest. Mech. Dyn. Astr., 100, 267-286, 2008.

[20] A. Rossi, G.B. Valsecchi, Collision risk against space debris in Earth orbits, Celest. Mech. Dyn. Astron., 95, 345-356, 2006.

[21] S. Valk, A. Lemaître, Analytical and semi-analytical investigations of geosynchronous space debris with high area-to-mass ratios, Advances in Space Research, 41, 1077-1090, 2008.

[22] S. Valk, A. Lemaître, L. Anselmo, Semi-analytical investigations of high area-to-mass ratio geosynchronous space debris including Earth's shadowing effects, Advances in Space Research, 42, 1429-1443, 2008.

[23] S. Valk, N. Delsate, A. Lemaître, T. Carletti, Global dynamics of high area-to-mass ratios geosynchronous space debris by means of the MEGNO indicator, Advances in Space Research, 43, 1509-1526, 2009.

[24] S. Valk, A. Lemaître, F. Deleflie, Semi-analytical theory of mean orbital motion for geosynchronous space debris under gravitational influence, Advances in Space Research, 43, 1070-1082, 2009.

[25] H.G. Walter, Conversion of osculating orbital elements into mean elements, Astr. J., 72, n. 8, 994-997, 1967.

[26] XMM-Newton Orbit and Constraints Details, http : //xmm2.esac.esa.int/external/xmm_sched/ vischeck/orbit_details.shtml

Department of Mathematics, University of Roma Tor Vergata, Via della Ricerca Scientifica 1, 00133 Roma (Italy)

E-mail address: celletti@mat.uniroma2.it

Department of Mathematics, Al. I. Cuza University, Bd. Carol I 11, 700506 Iasi (ROMANIA)

E-mail address: cgales@uaic.ro 